\section{Whole-exome sequencing reveals germline-mutated small cell lung cancer subtype with favorable response to DNA repair-targeted therapies}

\author{
Camille Tlemsani ${ }^{1{ }^{*}}{ }^{\dagger}$, Nobuyuki Takahashi ${ }^{1}$, Lorinc Pongor ${ }^{1}$, Vinodh N. Rajapakse ${ }^{1}$, Manoj Tyagi ${ }^{2 \dagger}$, \\ Xinyu Wen ${ }^{2}$, Grace-Ann Fasaye ${ }^{2}$, Keith T. Schmidt ${ }^{3}$, Parth Desai ${ }^{1}$, Chul Kim ${ }^{4}$, Arun Rajan ${ }^{5}$, \\ Shannon Swift ${ }^{5}$, Linda Sciuto ${ }^{1}$, Rasa Vilimas ${ }^{1}$, Santhana Webb ${ }^{1}$, Samantha Nichols ${ }^{1}$, \\ William Douglas Figg ${ }^{3}$, Yves Pommier ${ }^{1}$, Kathleen Calzone ${ }^{2}$, Seth M. Steinberg ${ }^{6}$, Jun S. Wei ${ }^{2}$, \\ Udayan Guha $^{5 \ddagger}$, Clesson E. Turner ${ }^{7}$, Javed Khan ${ }^{2}$, Anish Thomas ${ }^{1 \S}$
}

\begin{abstract}
Because tobacco is a potent carcinogen, secondary causes of lung cancer are often diminished in perceived importance. To assess the extent of inherited susceptibility to small cell lung cancer (SCLC), the most lethal type of lung cancer, we sequenced germline exomes of 87 patients ( 77 SCLC and 10 extrapulmonary small cell) and considered 607 genes, discovering 42 deleterious variants in 35 cancer-predisposition genes among $43.7 \%$ of patients. These findings were validated in an independent cohort of 79 patients with SCLC. Loss of heterozygosity was observed in 3 of $14(21.4 \%)$ tumors. Identification of variants influenced medical management and family member testing in nine (10.3\%) patients. Unselected patients with SCLC were more likely to carry germline RAD51 paralog $\mathrm{D}($ RAD51D), checkpoint kinase 1 (CHEK1), breast cancer 2 (BRCA2), and mutY DNA glycosylase (MUTYH) pathogenic variants than healthy controls. Germline genotype was significantly associated with the likelihood of a first-degree relative with cancer or lung cancer (odds ratio: $1.82, P=0.008$; and $2.60, P=0.028$ ), and longer recurrence-free survival after platinum-based chemotherapy $(P=0.002)$, independent of known prognostic factors. Treatment of a patient with relapsed SCLC and germline pathogenic mutation of $B R C A 1$ interacting protein C-terminal helicase 1 (BRIP1), a homologous recombination-related gene, using agents synthetically lethal with homologous recombination deficiency, resulted in a notable disease response. This work demonstrates that SCLC, currently thought to result almost exclusively from tobacco exposure, may have an inherited predisposition and lays the groundwork for targeted therapies based on the genes involved.
\end{abstract}

\section{INTRODUCTION}

Lung cancer is the most common cause of cancer death in the United States and worldwide, accounting for $18 \%$ of all cancer deaths and an estimated 1.7 million deaths each year (1). The most common etiology of lung cancer is tobacco smoking, accounting for more than $80 \%$ of cases. Although cigarette smoking is the main cause of lung cancer, not everyone who smokes develops lung cancer. About 10 to $15 \%$ of all lung cancers arise in never-smokers, implicating host genetic factors in lung cancer susceptibility. Genome-wide association studies have identified polymorphic variations in several candidate genes and loci as determinants of lung cancer risk (2). However, none of these genes are associated with large risks, although many are of considerable interest.

Of the major lung cancer histologies, small cell lung cancer (SCLC) - which accounts for nearly $13 \%$ of all lung cancer (3)-has

\footnotetext{
'Developmental Therapeutics Branch, Center for Cancer Research, NCl, Bethesda, MD 20892, USA. ${ }^{2}$ Genetics Branch, Center for Cancer Research, NCl, Bethesda, MD 20892, USA. ${ }^{3}$ Genitourinary Malignancies Branch, Center for Cancer Research, NCl, Bethesda, MD 20892, USA. ${ }^{4}$ Georgetown University, Washington, DC 20007, USA. ${ }^{5}$ Thoracic and GI Malignancies Branch, Center for Cancer Research, NCl, Bethesda, MD 20892, USA. ${ }^{6}$ Biostatistics and Data Management Section, Center for Cancer Research, NCl, Bethesda, MD 20892, USA. ${ }^{7}$ Walter Reed National Military Medical Center, Bethesda, MD, Bethesda, MD 20814, USA.

*Present address: INSERM, U1016, Institute Cochin, 75014 Paris, France.

†Present address: Laboratory of Pathology, Center for Cancer Research, NCl, Bethesda, MD 20892, USA.

¥Present address: Bristol-Myers Squibb, Lawrenceville, NJ 08648, USA.

§Corresponding author. Email: anish.thomas@nih.gov
}

the strongest association with smoking (4). It is also the most lethal. In most patients, SCLC has already metastasized outside the chest at diagnosis. The median survival of patients with SCLC is about 7 months and, owing to lack of effective therapies, has not substantially improved in decades (5). Even early detection by screeningwhich allows curative interventions in most other cancers-does not alter the clinical course of SCLC (6).

Tobacco smoking being a potent carcinogen, together with the observation that patients with SCLC are rarely never-smokers $(7,8)$, has influenced the prevailing notion that SCLC is a smoker's disease. Although case reports and small series have implicated tumor protein 53 (TP53) (9), RB transcriptional corepressor 1 (RB1) (10), and selected breast cancer 2 (BRCA2) variants (11), the germline risk for SCLC has not been investigated using comprehensive genomic approaches to date. SCLC was not represented in The Cancer Genome Atlas, which reported pathogenic or likely pathogenic germline variants in 2.2 to $22.9 \%$ of more than 10,000 patients across 33 cancers (12). Hence, little is known of a possible hereditary predisposition to SCLC.

Knowledge of the genes responsible for inherited susceptibility could be important for personalized therapies, as demonstrated by the approval of drugs targeting the DNA repair enzyme poly (adenosine 5'-diphosphate-ribose) polymerase 1 (PARP1) in patients with pancreatic, prostate, breast, and ovarian cancers that harbor defects in homologous recombination arising from $B R C A 1, B R C A 2$, or partner and localizer of $B R C A 2$ mutations. Identification of germline mutations could also prove useful for patients and families 
Table 1. Patient characteristics. Data are presented as $n$ (\%) or median (range). NA, not assessed; VALG, Veterans Administration Lung Study Group.

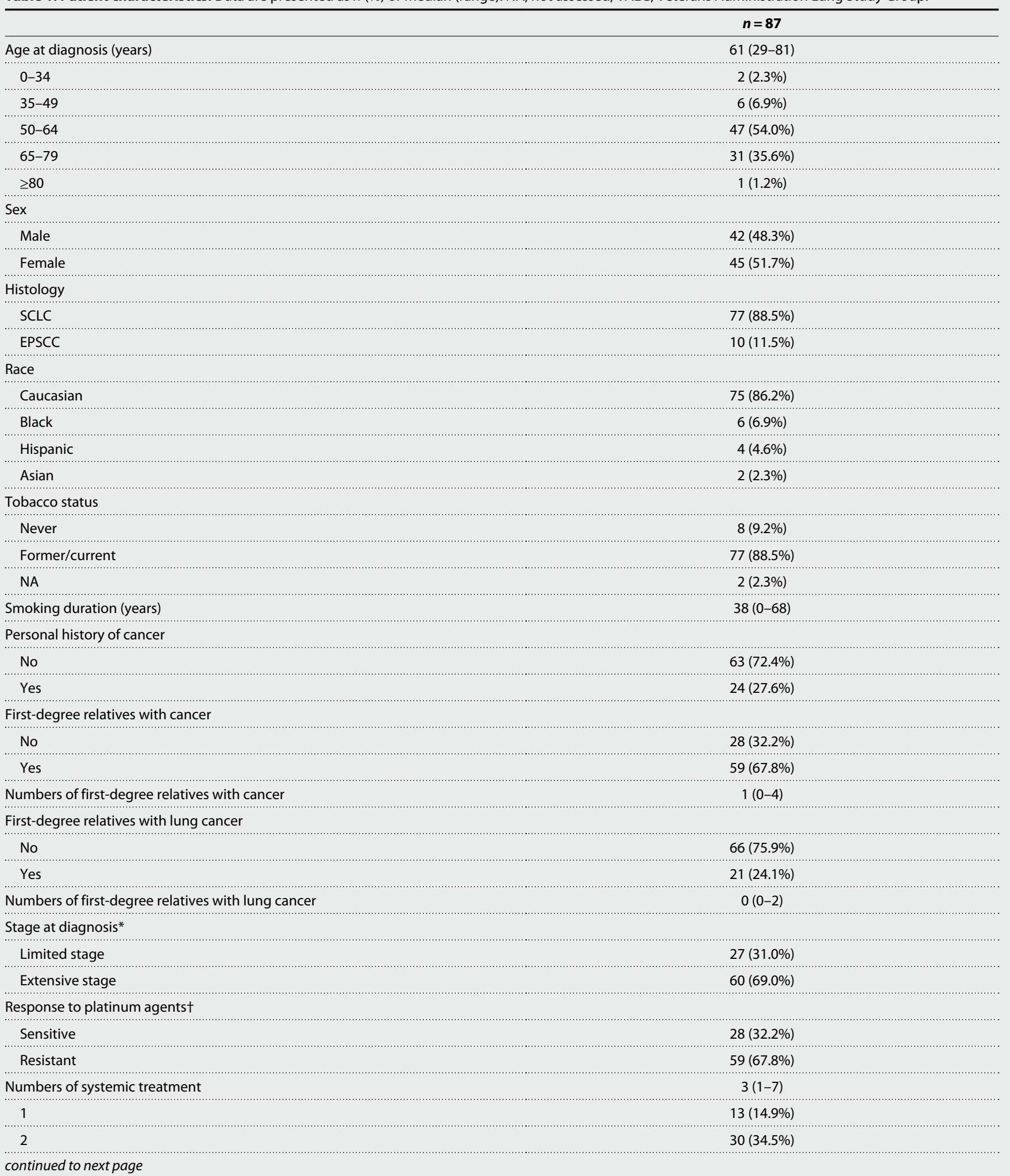


$n=87$

3

4

$\geq 5$
25 (28.7\%)

$10(11.5 \%)$

$9(10.3 \%)$

*The stage of patients with SCLC was defined according to the VALG stage. Among patients with EPSCC, localized disease was defined as limited stage and metastatic disease was defined as extensive stage. TPlatinum-sensitive was defined as disease progression $\geq 90$ days after first-line platinum-based chemotherapy, and platinum-resistant as disease progression $<90$ days or during first-line chemotherapy.

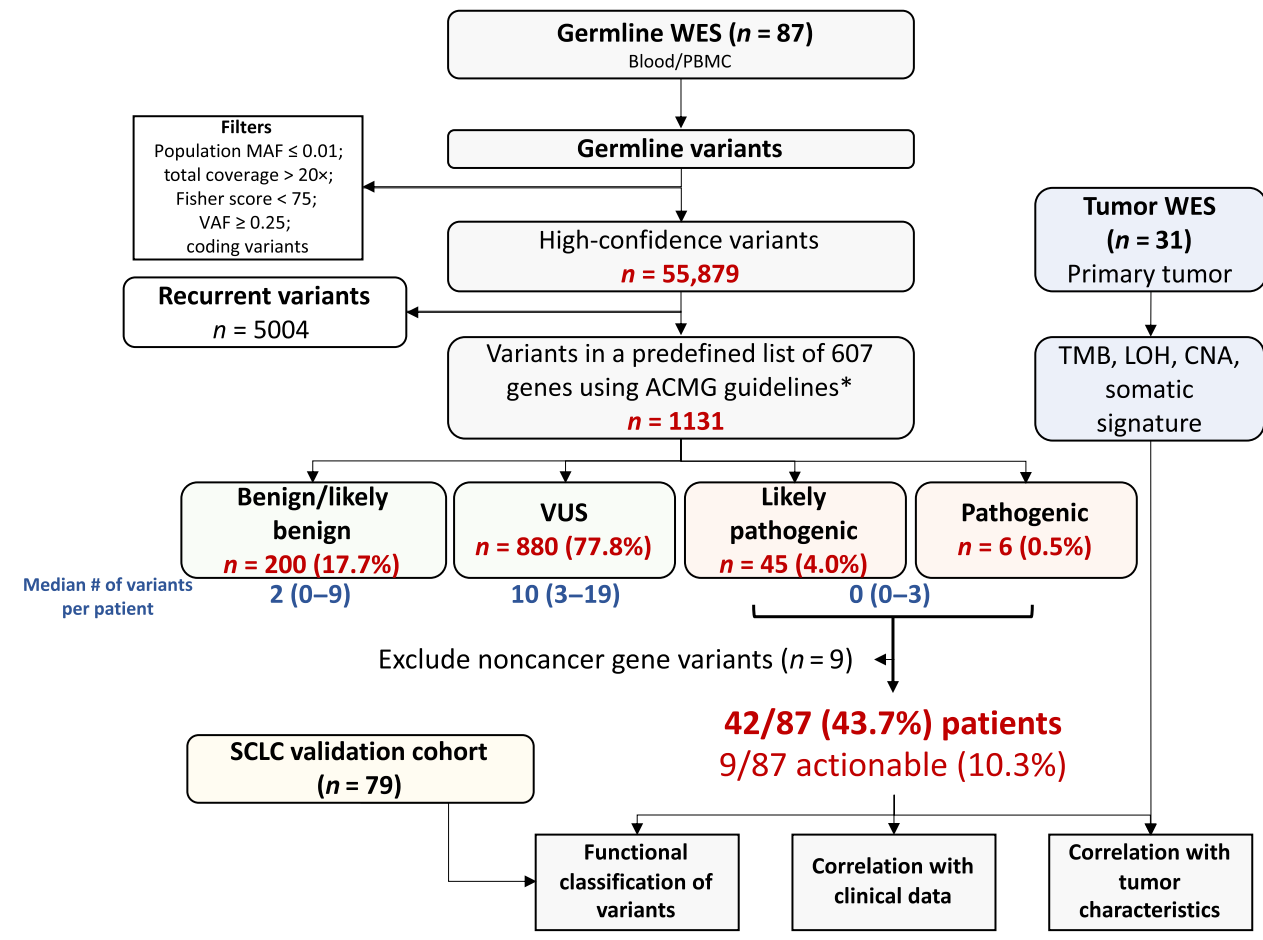

Fig. 1. Overview of the strategy to identify candidate SCNC cancer susceptibility genes. Eighty-seven patients with small cell neuroendocrine cancer (SCNC) were included. The characteristics of the patients are summarized in Table 1. Germline and tumor samples were whole exome-sequenced and aligned to human genome hg19 before variant calling and annotations. All germline variants were identified and filtered by (i) population minor allele frequency (MAF), (ii) total coverage $>20 \times$, (iii) Fisher score $<75$, (iv) $V A F \geq 0.25$, and (v) coding variants. We obtained a total of 55,879 variants. Among them, 1131 variants, belonging to a predefined list of 607 genes, were annotated as pathogenic, likely pathogenic, variant of uncertain significance (VUS), likely benign, or benign according the ACMG guidelines. The 607-gene list is provided in fig. S1 and table S3. For 31 available tumor samples, tumor mutation burden (TMB), loss of heterozygosity ( $\mathrm{LOH})$, copy number alteration (CNA), and mutational signatures were determined. We excluded nine likely pathogenic or pathogenic variants in noncancer-related genes [GAA, MYBPC3, FAH, COL7A1, ABCB11 (two variants), PAH, POLM, and PAH]. Only deleterious variants in cancer-related genes ( $n=42$ pathogenic or likely pathogenic variants in 87 patients) were included in the final analysis. Among them, 10 were actionable pathogenic variants. We also performed functional classification of variants and correlations with clinical characteristics of patients. Our data were validated with an independent small cell lung cancer (SCLC) cohort ( $n=79$ patients). VAF, variant allele frequency; WES, whole-exome sequencing.

for behavioral modification and complement cancer screening efforts. Extrapulmonary small cell cancers (EPSCC) are morphologically indistinguishable from SCLC (13) and share common molecular alterations and functional dependencies with SCLC (14). The molecular mechanisms underlying EPSCC development and progression remain poorly understood, and similar to SCLC, germline predisposition data are sparse.

To explore the genetic basis of SCLC and EPSCC and to identify candidate susceptibility genes, we performed whole-exome sequencing of germline DNA from 87 unselected patients and compared the frequency of pathogenic germline variants with 53,105 cancer-free non-Finnish European in dividuals from the Exome Aggregation Consortium (ExAC) cohort. The findings were validated in an independent SCLC cohort demonstrating that SCLC, one of the most lethal cancers, thought to result almost exclusively from tobacco use, may also have an inherited predisposition. Independent of known prognostic factors, germline genotype predicted favorable outcomes to DNA repair-targeted therapies, supporting the exploration of therapies that exploit synthetic lethality of DNA repair pathways in these patients.

\section{RESULTS \\ Study population}

A total of 87 patients with small cell neuroendocrine cancers (SCNCs; 77 patients with SCLC and 10 patients with EPSCC) were identified from among patients who attended the National Cancer Institute (NCI) Developmental Therapeutics Branch clinic. This included 68 consecutive patients who enrolled on a natural history study (ClinicalTrials.gov \#NCT02146170; NCI protocol \#14-C0105) offering germline sequencing and disclosure of results, and 19 patients identified using the National Institutes of Health Clinical Center's Biomedical Translational Research Information System (15). Patients were self-referred or were referred by treating physicians from across the United States. Patients were unselected for personal or family history of cancer, age at onset, stage of disease, or personal history of cancers. Tumor samples underwent central histopathologic review (table S1) confirming small-cell features.

The demographic characteristics of patients are summarized in Table 1. The median age at diagnosis was 61 years (range, 29 to 81 ). The majority of patients had SCLC $(n=77,88.5 \%)$. Other primary sites included bladder and cervix (2 patients each), larynx, rectum, 


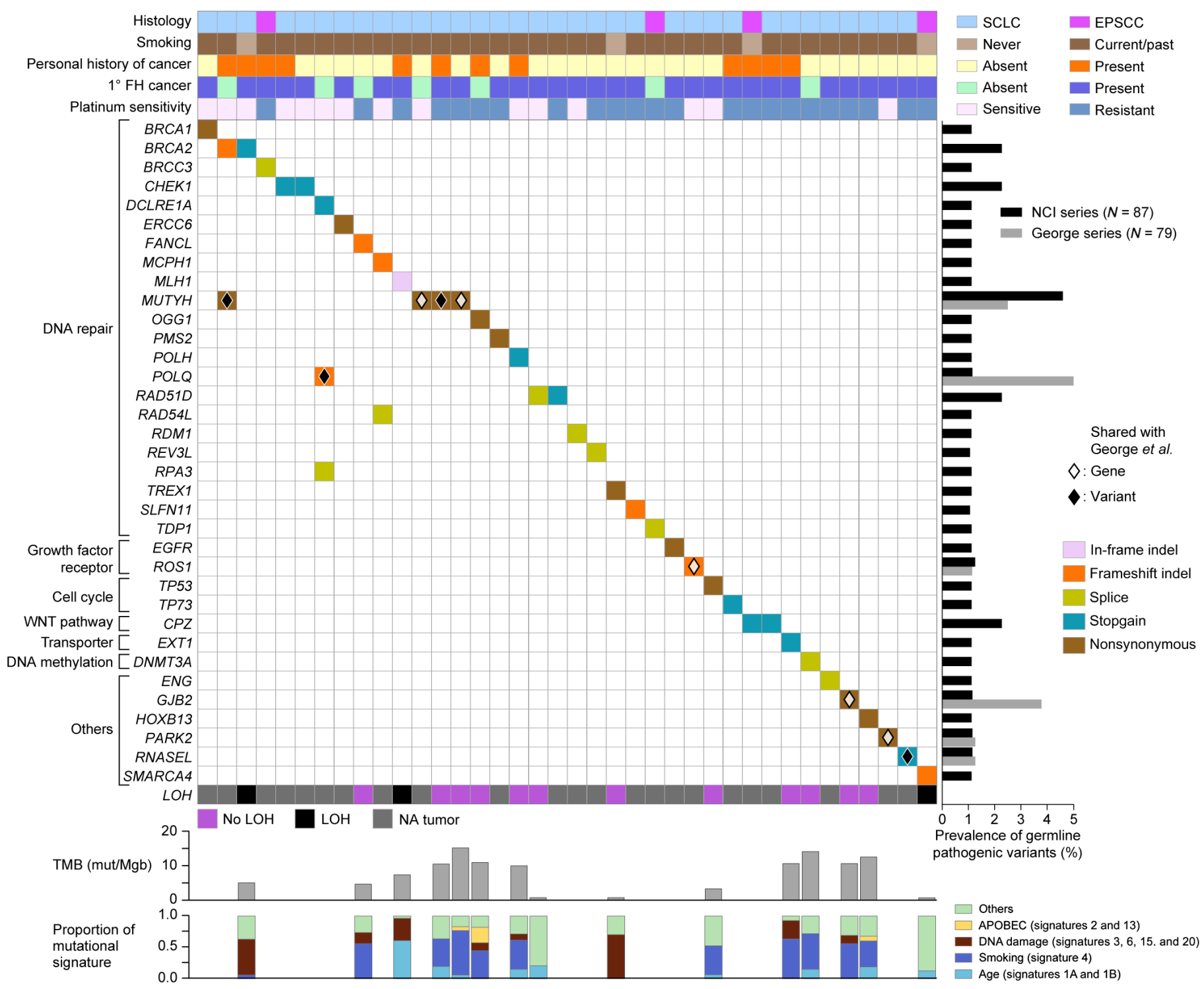

Fig. 2. Clinical and molecular characteristics of 42 patients with SCNC and pathogenic germline variants. Each column corresponds to a patient. The upper section shows the patient's clinical characteristics [histology, smoking history, personal history of cancer, first-degree relatives with cancer, and platinum sensitivity (platinumsensitive was defined as disease progression $\geq 90$ days after first-line platinum-based chemotherapy; platinum-resistant was defined as disease progression within 90 days or during first-line chemotherapy)]. APOBEC, apolipoprotein B mRNA-editing enzyme, catalytic polypeptide-like; EPSCC, extrapulmonary small cell cancer.

ovary, breast, gallbladder, and prostate (1 patient each). Most patients were current or former smokers $(n=77,88.5 \%)$. Eight $(9.2 \%)$ patients were never-smokers, of whom three had nonlung primary sites. Most patients $(n=59,67.8 \%)$ had a family history of firstdegree relative with cancer, and 21 patients $(24.1 \%)$ had family history of first-degree relative with lung cancer. All patients had received at least one previous systemic treatment. The duration of response to initial platinum-based therapy is associated with outcomes after second-line therapy for SCLC (16), and prognosis of patients with platinum-resistant disease is particularly worse. The majority of patients $(n=59,67.8 \%)$ in this study had platinum-resistant disease.

\section{Sequencing and germline pathogenic variants}

Germline whole-exome sequencing generated a median of 98 million reads of data per patient (range, 8 million to 173 million reads), resulting in a median of $96.6 \%$ (range, 92.3 to $98 \%$ ) and $90 \%$ (range, 31.7 to $95.3 \%$ ) bases covered at least 10 and 50 times, respectively (table S2).

We applied filters for quality and rarity in population databases to identify high-confidence coding variants and then excluded recurrent variants in this dataset (Fig. 1). We conducted in-depth manual analyses of variants in 607 genes that included (i) genes associated with hereditary cancers (both autosomal recessive and dominant transmission), (ii) cancer-associated genes (oncogenes and tumor suppressor genes based on Catalogue of Somatic Mutations in Cancer) (17), (iii) genes with recurrent somatic mutation in SCNCs (18), and (iv) 363 additional genes curated from previously published cancer predisposition genes (fig. S1 and table S3) $(18,19)$. Variants were classified as either benign and likely benign, variant of uncertain significance (VUS), or pathogenic and likely pathogenic 
Table 2. Molecular characteristics of pathogenic germline variants in patients with SCLC. VAF, variant allele frequency.

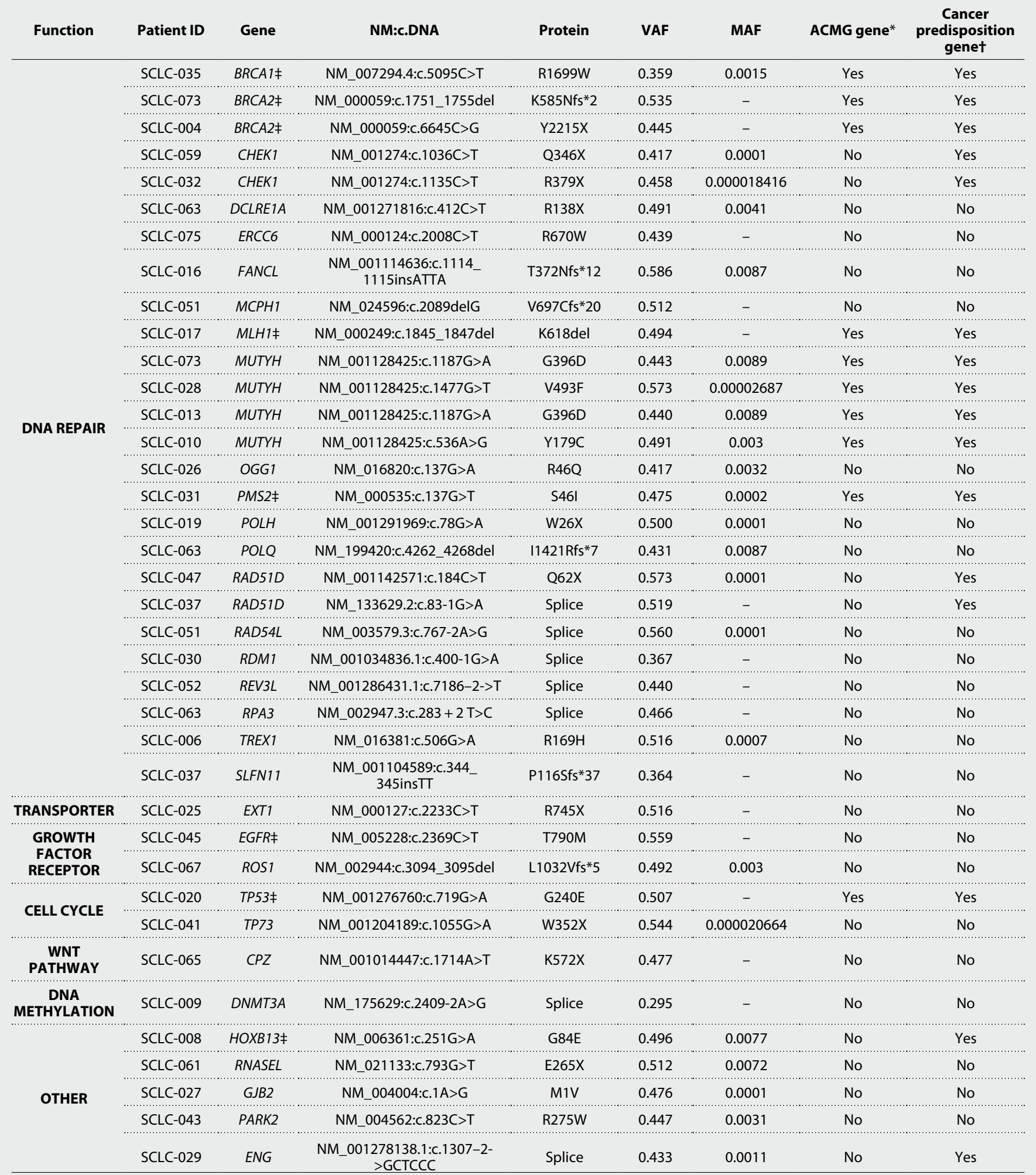

*ACMG gene = gene that belongs to the American College of Medical Genetics and Genomics (ACMG) published recommendations for reporting incidental findings. $\quad$ Cancer predisposition gene $=$ panel of 77 cancer predisposition genes (BROCA v10 panel). $\quad$ CConsidered actionable by a cancer geneticist. 
(deleterious) according to American College of Medical Genetics and Genomics (ACMG) recommendations (20). In 607 genes that were chosen for analyses, we identified 1131 nonsilent variants of which $6(0.5 \%)$ were deemed to be pathogenic, $45(4.0 \%)$ were likely pathogenic, $880(77.8 \%)$ were of uncertain significance, and 200 (17.7\%) were benign or likely benign. After excluding variants in noncancer genes, a total of 42 pathogenic or likely pathogenic variants were identified (table S4) in 35 genes, including $21.4 \%$ splice mutations, $26.2 \%$ stopgain mutations, $16.7 \%$ frameshift indels, $33.3 \%$ nonsynonymous mutations, and $2.4 \%$ nonframeshift indels. Most commonly mutated genes were related to DNA repair, accounting for $66.7 \%(n=28)$ of variants. The remaining genes were involved in growth factor receptor, cell cycle, Wnt pathway (4.8\%, two each), transporter, DNA methylation $(2.4 \%$, one each), and other pathways (14.3\%, six).

\section{Patients with pathogenic germline variants}

In the entire cohort, a total of 38 patients (43.7\%) carried pathogenic or likely pathogenic germline variants in 35 different cancer predisposition genes (Fig. 2 and Table 2). Three patients had more than one pathogenic germline variant detected (one patient with three variants and two patients with two variants each). On the basis of secondary review by a cancer geneticist (C.E.T.), nine patients (10.3\%) met criteria for clinical genetics referral; in other words, the germline genotype influenced medical management decisions and testing of at-risk family members. In addition, $11.5 \%$ of patients in our cohort had deleterious variants in ACMG genes (21), 10.3\% of patients in ACMG cancer genes (21), and 19.5\% of patients had pathogenic variants in BROCA v.10 genes (figs. S2 and S3) (22). The ACMG cancer genes consist of 24 high-penetrance autosomal dominant or recessive genes, whereas the BROCA panel includes 77 genes associated with hereditary cancer predisposition.

Of 77 patients with SCLC, 34 patients (44.2\%) carried pathogenic or likely pathogenic germline variants in 32 cancer predisposition genes (Fig. 2, Table 2, and figs. S2 and S4). Overall, the mutation categories, functions of genes affected, and proportion of patients with deleterious variants were similar to the overall cohort. Among the 10 patients with EPSCC, 4 patients harbored germline pathogenic variants in switch/sucrose non-fermentable-related, matrix-associated, actin-dependent regulator of chromatin, subfamily A, member 4 (SMARCA4), tyrosyl-DNA phosphodiesterase 1, BRCA1/BRCA2containing complex subunit 3, and carboxypeptidase Z. SMARCA4 mutation was detected in a 36-year-old woman with small cell carcinoma of the ovary, an aggressive monogenic cancer known to be driven by SMARCA4 mutations (23). Accordingly, her tumor displayed SMARCA4 loss of heterozygosity ( $\mathrm{LOH})$ and a low tumor mutation burden (TMB) of 0.84 mutations per megabase (mut/Mb).

We found pathogenic or likely pathogenic variants in $B R C A 2$ in two patients and in BRCA1, TP53, homeobox B13 (HOXB13), epidermal growth factor receptor (EGFR), Parkinson disease 2 (PARK2), PMS1 homolog 2 (PMS2), and mutL homolog 1 (MLH1) in one patient each (Fig. 2 and Table 2). The $M L H 1$ germline variant (c.1845_1847del, p.K618del), reported as pathogenic by multiple independent ClinVar submitters, was observed in a 65-year-old ex-smoker with history of uterine cancer at age 48 and a family history of Lynch syndrome-related cancers (Fig. 3, A to C). The patient's tumor exhibited relatively low MSIsensor score at 2.2 (24) but harbored exceptionally high insertion/deletions (total number of insertions and deletions: 129), indicating microsatellite instability. Furthermore, the tumor showed $M L H 1 \mathrm{LOH}$, absence of MLH1
RNA and protein expression (Fig. 3D and fig. S5), and mutational signature pattern (25) consistent with defective DNA mismatch repair (signatures 6 and 15) (Fig. 3E), together supporting the role of $M L H 1$ mutation in SCLC susceptibility. One of two patients with deleterious BRCA2 germline variant (Fig. 3F) was a 67-year-old never-smoker. The BRCA2 variant displayed somatic $\mathrm{LOH}$ and loss of RNA expression (Fig. 3, G and H), with evidence of chromosomal instability (Fig. 3I) and predominance of mutational signature associated with failure of DNA double-strand break repair by homologous recombination (Fig. 3J).

\section{SCLC tumor characteristics}

Matched tumor and normal exome sequencing were available for 31 patients (27 SCLC and 4 EPSCC), including 14 (45.2\%) patients with germline deleterious variants. The median tumor purity across all samples was $81 \%$ (range, 23 to 98 ) (table S5). LOH was observed in 3 of $14(21.4 \%)$ tumors in patients with germline deleterious $B R C A 2, M L H 1$, and SMARCA4 mutations, suggesting that the variants are undergoing positive selection in the context of the classic two-hit model (Fig. 2A) (26). Somatic mutations or LOH were not found in other tumors. TMB was not significantly different between patients with and without germline pathogenic variants [median (range) TMB, $10.1 \mathrm{mut} / \mathrm{Mb}(0.3$ to 15.3$)$ and $5.0 \mathrm{mut} / \mathrm{Mb}(0.7$ to $14.3, P=0.35)$ ] (fig. S6A). Tumors of patients with germline pathogenic variants in DNA repair genes did not have a significantly different proportion of mutational signatures associated with DNA repair defects (signatures $3,15,16$, and 20) compared with those without (median total number of mutations: 358.1 versus 91.7 mutations, $P=0.30$ ) (fig. $\mathrm{S} 6 \mathrm{~B}$ ). Mutational signature contributions for each sample are detailed in fig. S6C.

\section{SCLC independent cohort}

We compared our findings with whole-genome sequencing data from an independent cohort of 79 patients with SCLC from George et al. (18) using the same filters as in our study (fig. S2 and table S6). Unlike our cohort where most patients had metastatic and recurrent SCLC, the independent cohort consisted mostly of patients with early-stage SCLC who underwent surgical resection of their primary tumor. Somatic mutation characteristics of both cohorts are summarized in fig. S7. A total of 40 pathogenic germline variants were identified among 33 patients. The frequency of pathogenic germline variants in the independent cohort mirrored our cohort ( $40.2 \%$ of patients versus $44.2 \%$ in our cohort; Fig. $4 \mathrm{~A}$ ). The overall mutation categories and functions of genes affected were also similar between the two cohorts (Fig. 4, B and C). Several genes [mutY DNA glycosylase (MUTYH), ROS Proto-Oncogene 1 (ROS1), DNA polymerase theta (POLQ), ribonuclease L (RNASEL), PARK2, and gap junction protein beta 2 (GJB2)] and variants (MUTYH G396D, POLQ I1421Rfs*7, and RNASEL E265X) were recurrently mutated in both cohorts (Fig. 2 and fig. S8).

\section{Comparison of mutation frequency with the population}

To assess the frequencies of pathogenic germline variants detected in our cohort in comparison to the general population, we analyzed the ExAC cohort of cancer-free individuals ( $n=53,105$ individuals) (27). The variants were rare and found in less than $1 \%$ of the individuals examined, across all ethnicities (table S7). Using a $P<0.05$, our cohort showed a higher than expected rate of pathogenic variants in the RAD51 paralog $\mathrm{D}(R A D 51 D)$, checkpoint kinase 1 (CHEK1), BRCA2, and MUTYH genes (Fig. 4, D and E). MUTYH, 


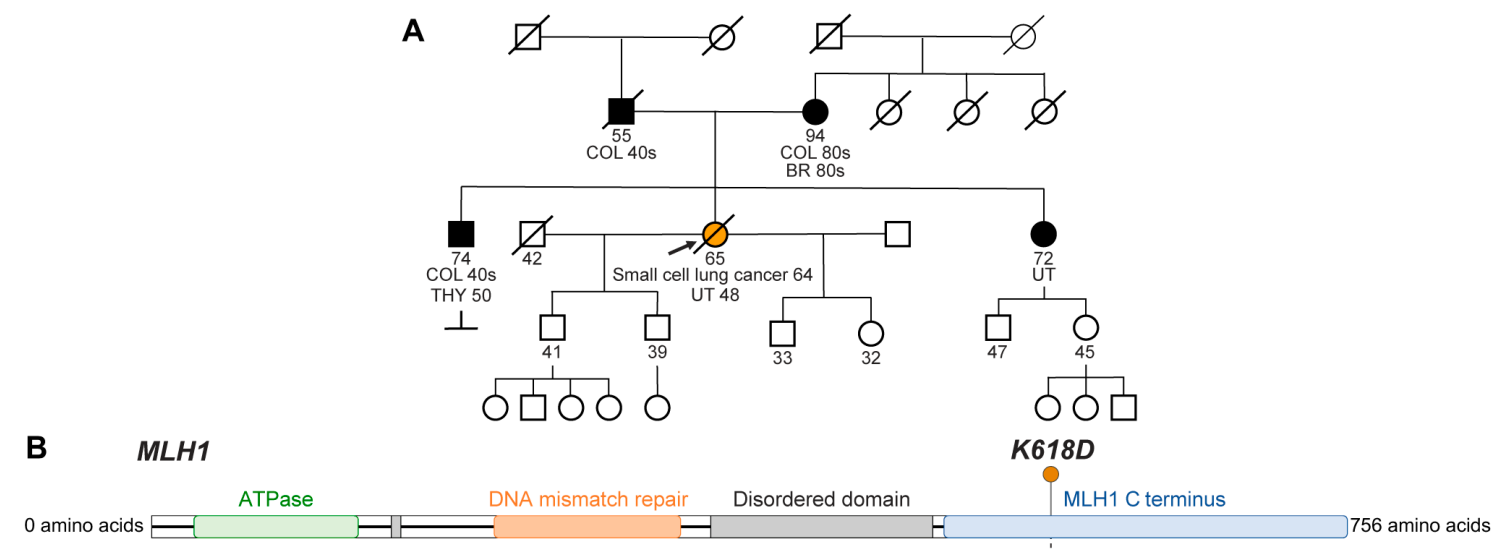

C

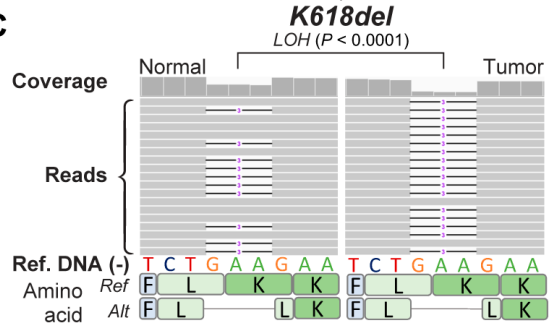

D

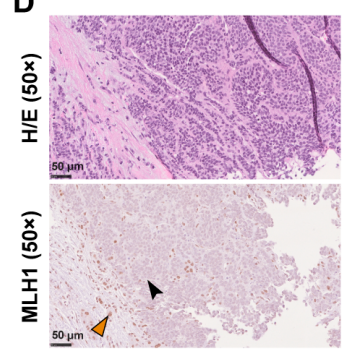

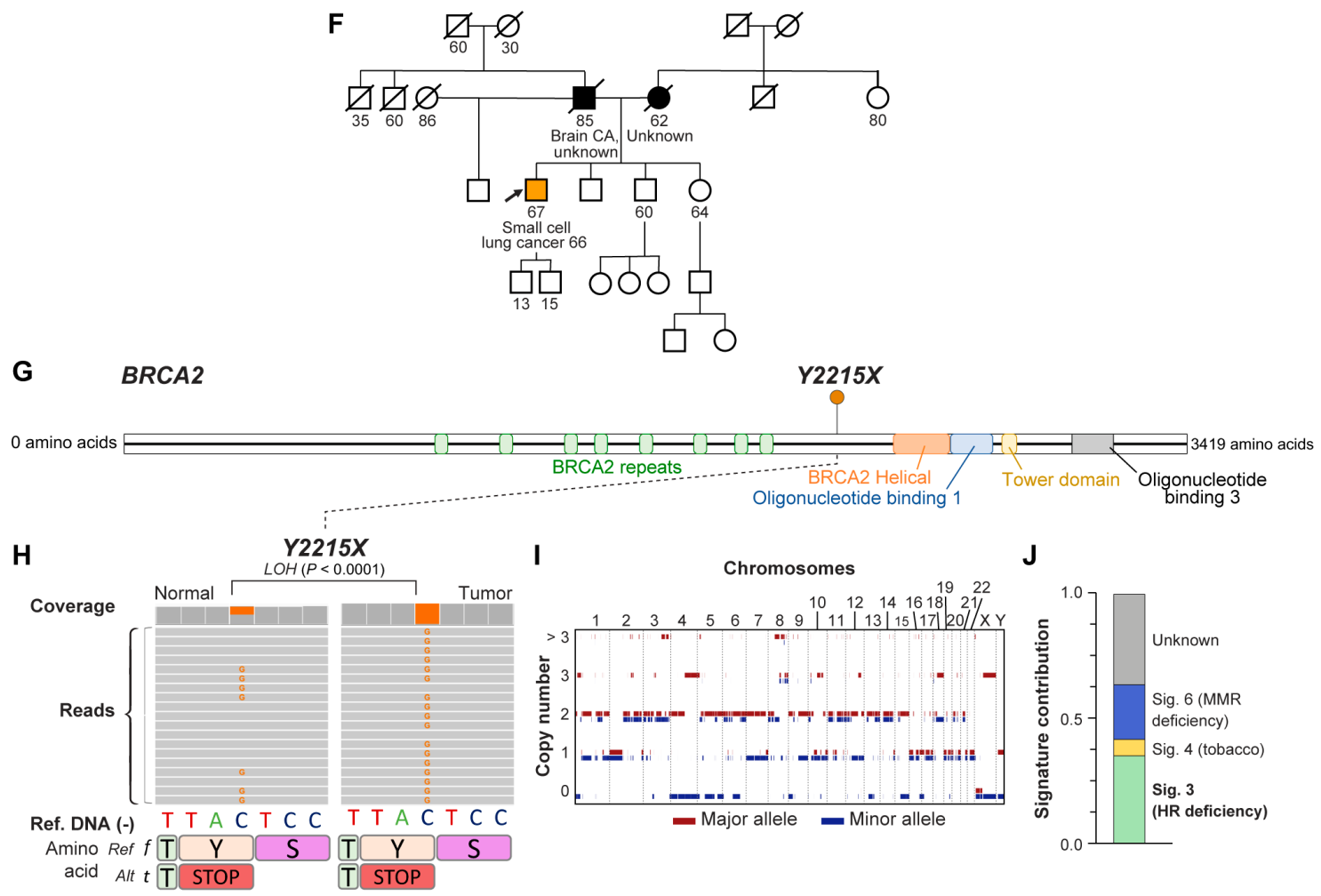

E

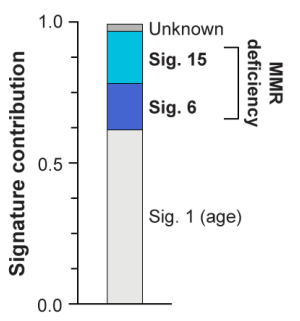

Fig. 3. Actionable pathogenic germline variants: Examples of $\mathbf{M L H} 1$ and BRCA1 in patients with SCLC. (A) Genogram of the MLH1 pathogenic variant carrier (SCLC017). Family members with SCLC and other cancers are shown in orange and black, respectively. (B) Lolliplot of MLH1 pathogenic germline variant. (C) Integrative genomics viewer (IGV) screenshots of MLH1 pathogenic variant. (D) Immunohistochemistry showing hematoxylin and eosin (H\&E) (top) and MLH1 protein expression (bottom) in liver metastasis. Loss of MLH1 nuclear staining seen in tumor cells (black arrowhead) but not in normal hepatic cells (orange arrowhead). (E) Mutational signature proportion in MLH1 germline-mutated tumor sample. MMR, mismatch repair. (F) Genogram of BRCA2 pathogenic variant carrier (SCLC-004). Family members with SCLC and other cancers shown in orange and black, respectively. (G) Lolliplot of BRCA2 pathogenic germline variant. (H) IGV screenshot of $B R C A 2$ pathogenic variant. (I) Copy number variation profile and $(\mathbf{J})$ mutational signature proportion in BRCA2 germline-mutated tumor. COL, colorectal cancer; BR, breast cancer; THY, thyroid cancer; UT, endometrial cancer. 

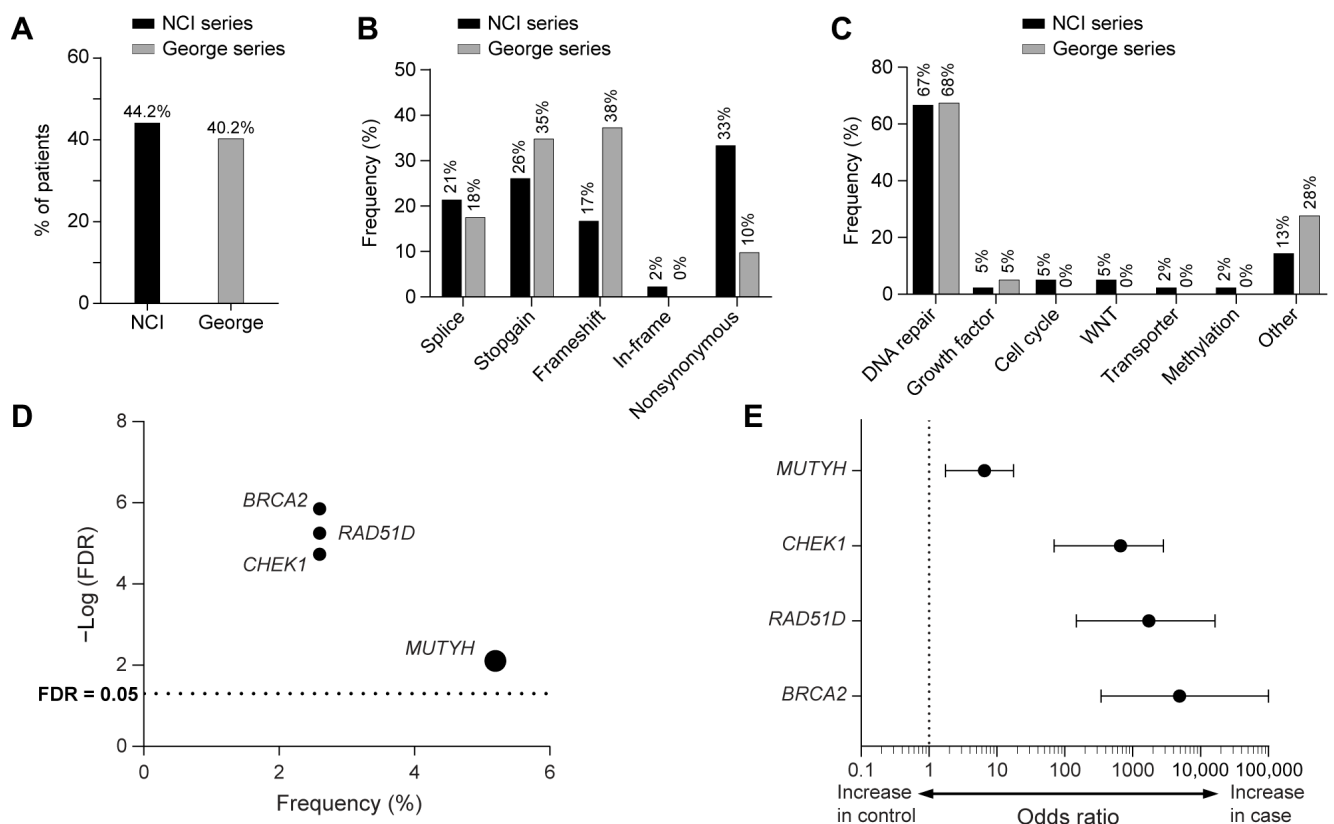

E
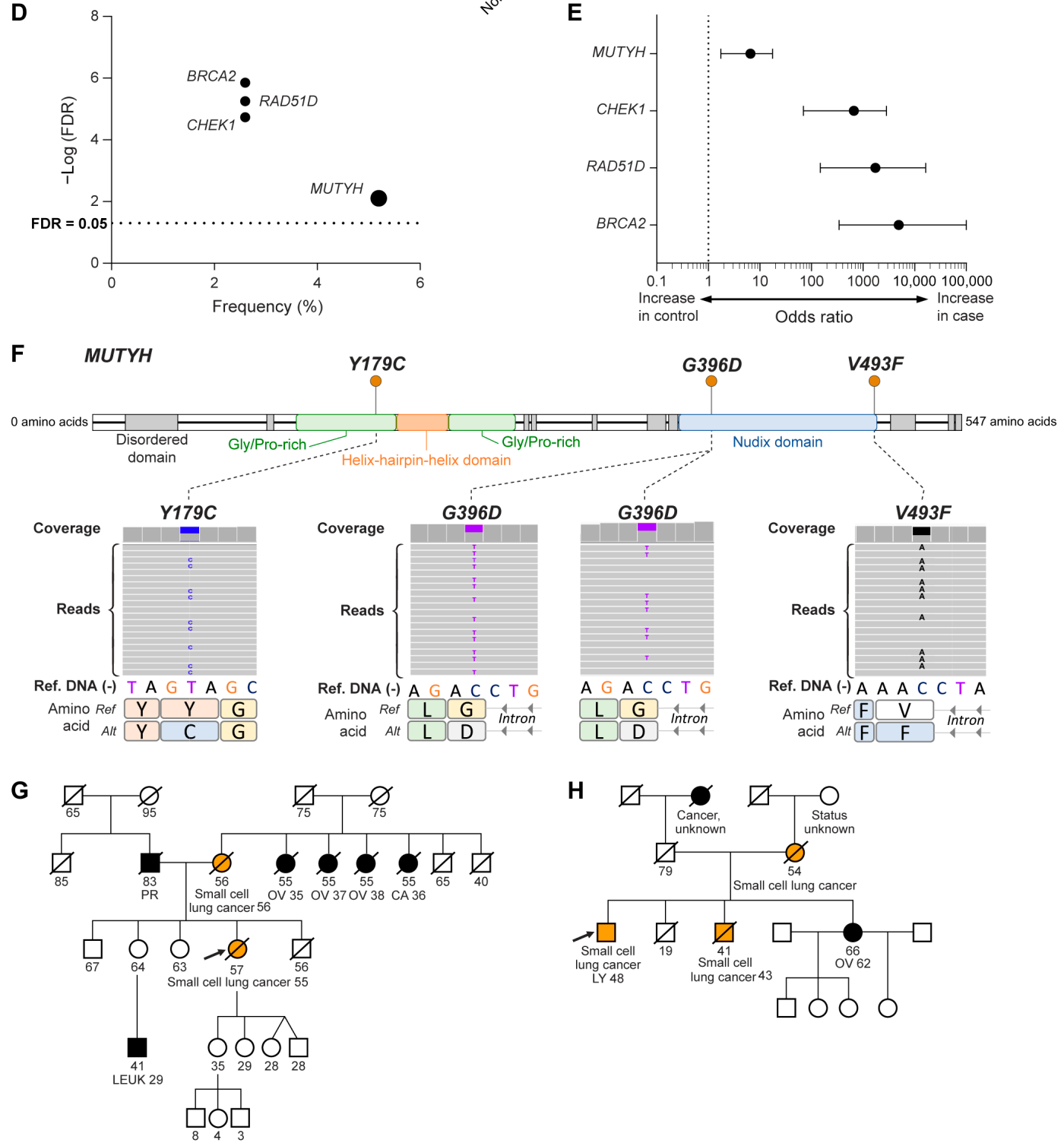

Fig. 4. Pathogenic germline variants in SCLC: Frequency in an independent cohort, enrichment relative to healthy controls, and family history of SCLC in MUTYH variant carriers. (A) Overall proportion of pathogenic germline variants and variants according to (B) gene categories and (C) gene function among patients with SCLC in our cohort (in black) and in the independent SCLC cohort [George et al. (18); in gray]. The numbers show proportion of patients with pathogenic germline variants of indicated gene categories and function in each cohort. (D) Gene-based enrichment analysis of the pathogenic alterations in our cohort relative to 53,105 cancer-free ancestry-matched individuals from the Exome Aggregation Consortium (ExAC) cohort. The $x$ axis represents the frequency of each significantly mutated gene and the $y$ axis the - $\log$ (FDR). FDR (false discovery rate) represents the $P$ value adjusted for FDR. We chose FDR $<0.05$ as a cutoff for significance. (E) Pathogenic alterations enriched in our cohort compared with the ExAC cohort. Error bars indicate $95 \%$ confidence intervals (Cls). (F) Lolliplot of MUTYH gene showing pathogenic germline variants found in our cohort (SCLC-010, 013, 028, and 074) and IGV screenshots of the four MUTYH pathogenic variants found in our cohort. (G and H) Genograms of two MUTYH pathogenic variant carriers in our cohort [SCLC-010 in (G) and SCLC-013 in (H)]. Family members with SCLC and other cancers shown in orange and black, respectively. OV, ovarian cancer; PR, prostate cancer; LEUK, leukemia (unknown subtype); LY, lymphoma (unknown subtype). 
A

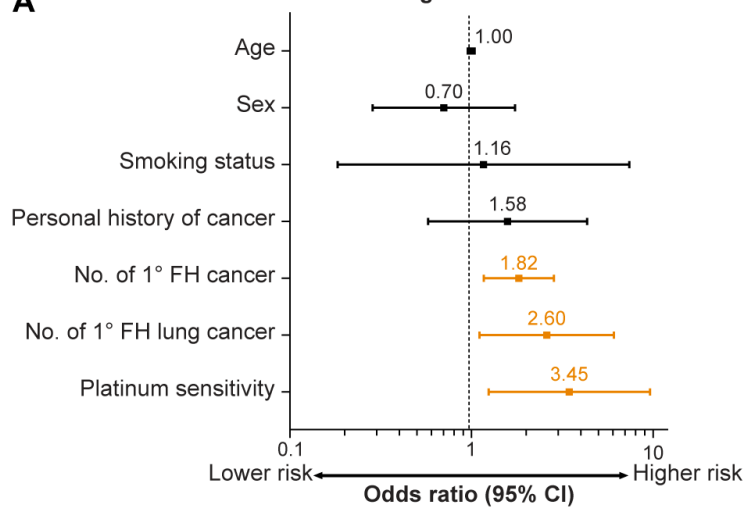

B

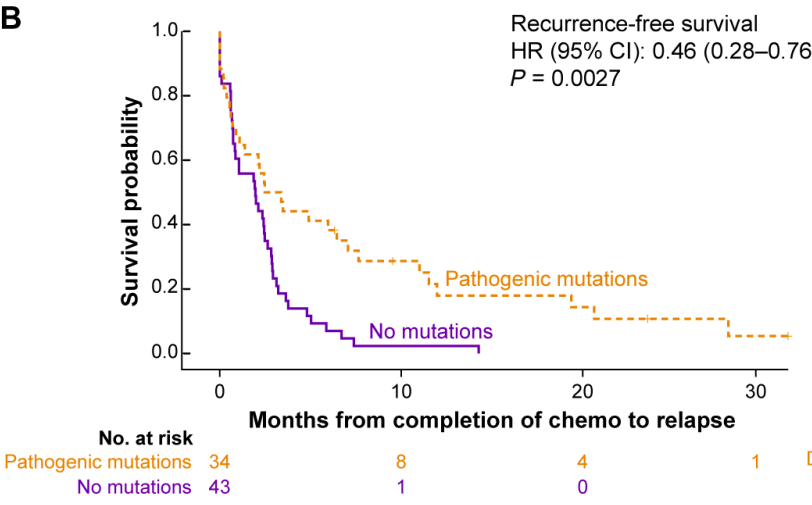

C

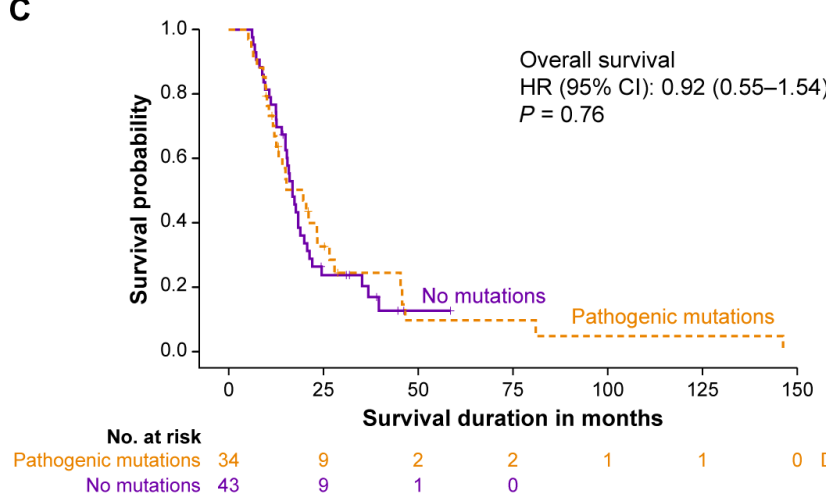

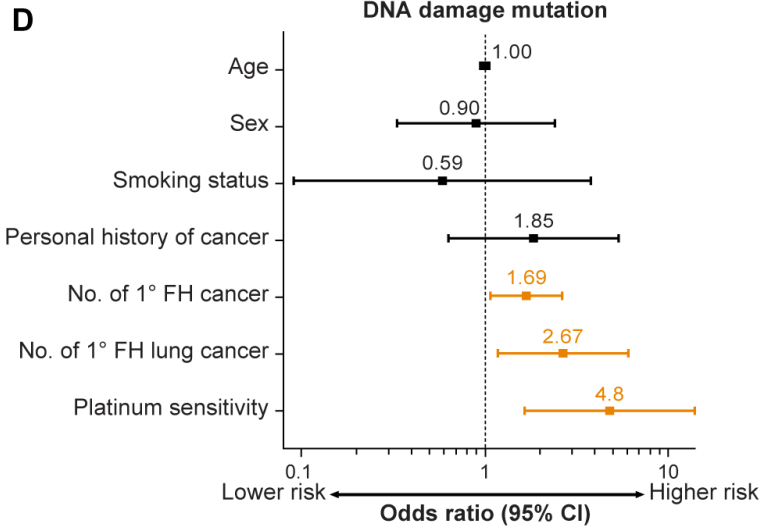

E

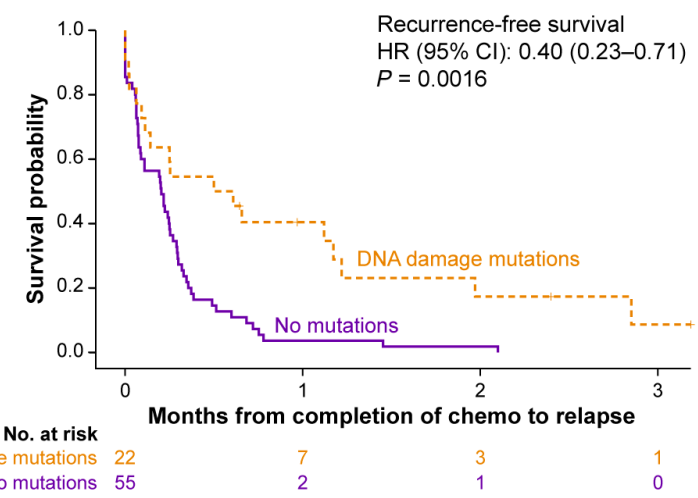

$\mathbf{F}$

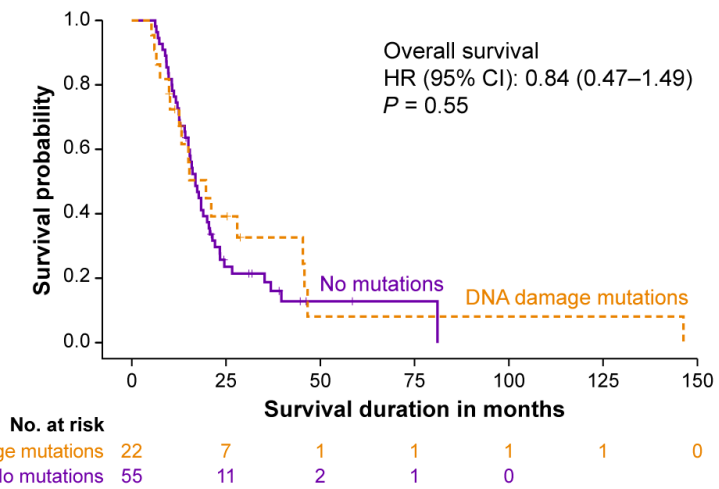

Fig. 5. Clinical characteristics and germline mutations. (A) Risk of germline mutations in SCLC based on clinical characteristics. References of categorical values $(\mathrm{OR}=1)$ : sex: male; smoking status: never smoker; personal history of cancer: no personal history of cancer; platinum sensitivity: platinum-sensitive. Platinum-sensitive was defined as disease progression $\geq 90$ days after first-line platinum-based chemotherapy, and platinum-resistant was defined as disease progression within 90 days or during first-line chemotherapy. $P$ values shown in the figures are for a test of hazard ratio (HR) $=1.0$ (univariate analysis). Kaplan-Meier curves of RFS (B) and OS (C) in patients with SCLC who have germline mutations versus those who do not. (D) Risk of pathogenic germline DNA damage variants in SCLC based on clinical characteristics. References of categorical values $(\mathrm{OR}=1)$ : sex: male; smoking status: never smoker; personal history of cancer: no personal history of cancer. $P$ values shown in the figures are for a test of $\mathrm{HR}=1.0$ (univariate analysis). Kaplan-Meier curves of RFS (E) and OS (F) in patients with SCLC who have DNA repair germline mutations versus those who do not. No. of $1^{\circ} \mathrm{FH}$ cancer, number of first-degree relatives with cancer; no. of $1^{\circ} \mathrm{FH}$ lung cancer, number of first-degree relatives with lung cancer; $95 \% \mathrm{Cl}$, $95 \%$ confidence interval.

which plays a major role in protecting against oxidative DNA damage and base excision repair $(28,29)$, was recurrently mutated in our cohort with four (5.2\%) affected patients, including two patients who harbored the same variant c.1187G >A, p.G396D (population minor allele frequency $=0.0089$ ) (Fig. $4 \mathrm{~F}$ ). The same $M U T Y H$ variant was also found in two patients in the independent cohort
(18), is known to be a founder mutation in European populations, and is enriched in patients with breast cancer (30). No second events were found at the somatic level among the four patients (two from our cohort and two from the independent cohort) with germline MUTYH deleterious variants and available somatic genomic data. MUTYH biallelic mutations underlie familial colorectal cancer, and 
A
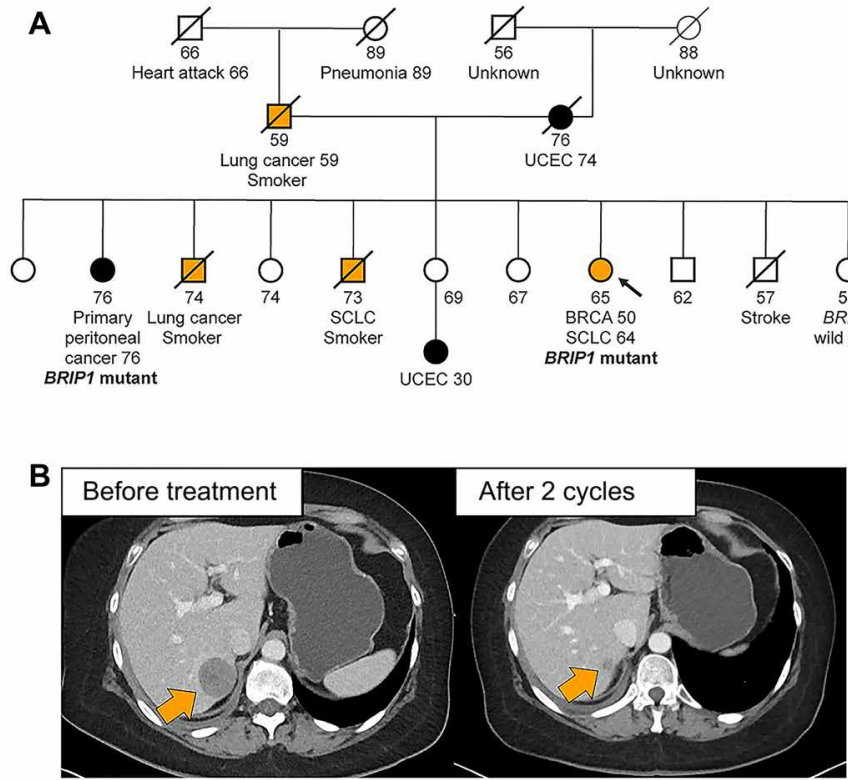

C

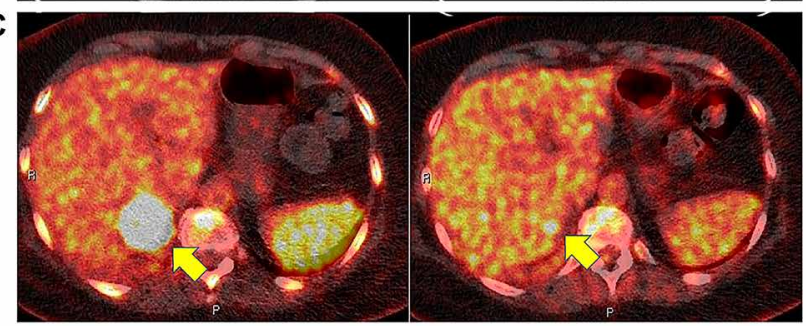

Fig. 6. Response to drug combination synthetically lethal with HRD in a patient with SCLC with pathogenic germline homologous recombination gene variant. (A) Genogram highlighting family history of endometrial cancers and lung cancers in the patient with SCLC and BRIP1 pathogenic germline variant (NM_032043.2: c.514A $>$ T; p.K172*). Family members with history of lung cancer are indicated in orange. Of two siblings who underwent germline testing, one with a personal history of primary peritoneal cancer carried the BRIP1 variant. $C$, computed tomography $(\mathbf{B})$ and fluorodeoxyglucose-positron emission tomography (FDG-PET) (C) imaging 2 months after starting nanoparticle camptothecin NLG207 $12 \mathrm{mg} / \mathrm{m}^{2}$ (every 2 weeks intravenously) and poly(adenosine 5 '-diphosphate-ribose) polymerase (PARP) inhibitor olaparib $250 \mathrm{mg}$ twice daily by mouth. The recurrent SCLC liver lesion markedly reduced in size (orange arrows) and metabolic activity (yellow arrows) in CT and FDG-PET scans, respectively. HRD, homologous recombination deficiency; UCEC, uterine corpus endometrial carcinoma; BRCA, breast cancer.

monoallelic MUTYH mutations confer a small increase in the risk of colorectal cancer (31). Although all variants in our cohort were heterozygous, MUTYH carriers had a strong family history of cancer, including multiple family members with SCLC (Fig. 4, G and H).

\section{Clinical characteristics of SCLC pathogenic germline variant carriers}

We assessed the association between pathogenic germline variants and clinical characteristics, treatment response, and survival (Fig. 5 and table S8). All patients received platinum-based chemotherapy as their first-line treatment (table S9). The median patient follow-up was 30.6 months. Patients with SCLC and pathogenic or likely pathogenic variants were more likely to have a first-degree relative with cancer and a first-degree relative with lung cancer \{odds ratio (OR) [95\% confidence interval (CI)]: 1.82 (1.17 to 2.84), $P=0.008$; and 2.60 (1.11 to 6.09 ), $P=0.028$, respectively\} compared with pa- tients without pathogenic variants (Fig. 5A). Presence of pathogenic germline variant was associated with a significantly higher likelihood of platinum sensitivity [OR (95\% CI): 3.45 (1.24 to 9.62), $P=0.018]$. Consistent with this finding, patients with a pathogenic variant had significantly longer recurrence-free survival [RFS, median (95\% CI): 3.0 months ( 0.9 to 7.2 )] than patients without [2.0 months ( 0.8 to 2.5$), P=0.0027$ by log-rank test] (Fig. $5 \mathrm{~B})$. However, no significant difference in overall survival (OS) was found between patients with a pathogenic variant [19.7 months (12.1 to 23.4)] and those without [median OS, 16.9 months (15 to 20), $P=0.76$ ] (Fig. 5C). In multivariable analysis (including age and stage at diagnosis, and sex), germline genotype was independently associated with better RFS $[P=0.003$, hazard ratio $(\mathrm{HR})=0.44$ ( $95 \%$ CI, 0.25 to 0.76$)]$.

Similarly, DNA repair gene pathogenic variant carriers were more likely to have a first-degree relative with cancer and a firstdegree relative with lung cancer [1.69 (1.08 to 2.65), $P=0.023$; and 2.67 (1.18 to 6.07), $P=0.019$, respectively] compared to noncarriers (Fig. 5D). The OR for platinum sensitivity was markedly higher for patients with DNA repair gene pathogenic variants [4.80 (1.65 to 13.97, $P=0.004)$ ] compared with patients with no DNA repair gene pathogenic variants. Patients with DNA repair gene pathogenic variants also had significantly longer RFS compared with those with no DNA repair gene pathogenic variants [median RFS $(95 \% \mathrm{CI})$ : 5.5 months ( 0.9 to 11.7 ) versus 2.0 months ( 0.8 to 2.5 ), $P=0.0016$ ] (Fig. 5E). No difference in OS was found between the two groups [median OS 19.7 months (10.1 to 45.4) and 16.9 months (14.2 to 20.5), $P=0.55$ ] (Fig. 5F). Multivariable analysis revealed that DNA repair gene germline pathogenic variants were independently associated with a better RFS $[P=0.0004, \mathrm{HR}=0.34(95 \% \mathrm{CI}, 0.19$ to 0.62$)]$.

Subgroup analyses stratifying patients by stage at diagnosis also demonstrated that patients with pathogenic or DNA repair gene germline variants often had improved RFS after platinum-based chemotherapy compared to those without (fig. S9). The impact of the germline genotype on improved RFS was significant when we considered only the BROCA cancer predisposition genes $[P=0.012$, $\mathrm{HR}=0.38$ (95\% CI, 0.18 to 0.81 ) ] (fig. S10), but not the ACMG genes $[P=0.059, \mathrm{HR}=0.44$ (95\% CI, 0.19 to 1.03$)]$ (fig. S11).

\section{Response to drug combination synthetically lethal with homologous recombination deficiency in a patient with SCLC and a pathogenic germline homologous recombination repair-gene variant}

The relevance of these observations for therapeutics was tested in a single patient with SCLC and germline pathogenic BRIP1 variant (NM_032043.2: c.514A>T; p.K172*). The patient presented with progressive disease after combination therapy with platinum, etoposide, and radiation after enrollment to the original cohort was completed. BRIP1 encodes a helicase protein that interacts with $B R C T$ repeats of $B R C A 1$ and is essential in the normal double-strand break repair function of the $B R C A 1$ complex. Monoallelic pathogenic germline mutations in BRIP1 confer a high risk of ovarian cancer (32). The patient had a strong family history of endometrial and lung cancers, and the patient's sister with a history of primary peritoneal cancer also harbored the same germline BRIP1 variant (Fig. 6A).

Given limited efficacy of monotherapies in SCLC, we considered a combination of two agents-a PARP inhibitor and a topoisomerase 1 (TOP1) inhibitor-both synthetically lethal with homologous recombination deficiency (HRD) (33). While PARP inhibitors were 
the first cancer therapeutics designed to exploit synthetic lethality $(34,35)$, it has long been known that yeast, which is intrinsically resistant to TOP1 inhibitors, becomes highly sensitive upon inactivation of key components of the homologous recombination machinery $(36,37)$. Furthermore, combinations of TOP1 and PARP inhibitors are highly synergistic in cell line and tumor models with HRD (38).

With this rationale, the patient was enrolled on a phase 2 clinical trial (ClinicalTrials.gov identifier: NCT02769962) combining camptothecin (formulated as a nanoparticle NLG207), a highly selective inhibitor of TOP1, and PARP inhibitor olaparib. The camptothecin/ PARP inhibitor combination resulted in substantial reduction in this patient's tumor burden and resolution of cancer pain (Fig. 6, B and C).

\section{DISCUSSION}

SCLC is an exceptionally aggressive and lethal form of lung cancer. Characterization of somatic genomes and transcriptomes has not provided the expected opportunities for targeted therapeutic interventions in this cancer (18), and SCLC is approached clinically as a single disease entity. Here, we report that a small yet an important proportion of patients with SCLC harbors deleterious germline variants in high and moderate penetrance genes. Inherited loss-of-function mutations in DNA repair genes $R A D 51 D, C H E K 1$, and $B R C A 2$ are more frequent among patients with SCLC than among controls. After adjusting for known prognostic factors, germline genotype was associated with favorable outcomes to DNA-damaging chemotherapies. These findings demonstrate that SCLC carcinogenesis is not only driven by tobacco exposure but may also be influenced by the germline genetic background. Our results define a subgroup of patients with SCLC in whom the germline genotype may inform prognosis and the choice of targeted therapies.

Cisplatin or carboplatin and etoposide form the backbone of current frontline standard-of-care therapy for SCLC. However, not all patients respond, and there are no biomarkers to identify likely responders. Our results-notable especially given the modest cohort size-demonstrate that, after adjusting for age, sex, and stage at diagnosis, patients with an inherited loss-of-function variant have more favorable responses to platinum-based chemotherapy. The effect of genotype was particularly notable for patients with DNA repair gene variants. Among patients with ovarian, breast, prostate, and pancreatic cancers, inherited and somatic mutations in DNA repair genes define a subset of tumors that are particularly sensitive to PARP inhibitors and platinum-based chemotherapy (39). The improved RFS of our patients mirrors that of patients with these other solid tumor subtypes and points to a similar DNA-damaging therapy-responsive subset among SCLC. Despite the RFS benefit, germline genotype was not associated with OS in the present study, likely reflecting the underlying resistance mechanisms. We observed a marked tumor regression to a combination of PARP inhibitor and TOP1 inhibitor in a patient with SCLC and a pathogenic germline BRIP1 variant. Although caution must be exercised in interpreting and extrapolating from the response of a single patient, the notable response to drugs synthetic lethal with DNA repair pathways provides compelling impetus to conduct rigorous clinical evaluation of the effect of such drugs on overall response rate and measures of survival in patients with SCLC selected for germline DNA repair defects.

Germline genotype may identify smokers at high risk of SCLC who could then be targeted for early interventions. Germline genotype may also help identify the patients and families who are susceptible to developing additional cancers and who could potentially benefit from genetic counseling and cancer prevention interventions. Age and tobacco smoking were not associated with pathogenic germline variants in our cohort. Rather, we found an enrichment of first-degree family members with cancers among the pathogenic germline variant carriers. Further studies will be needed to determine whether family history-based criteria can identify the carriers.

This study had limited ability to assign causality to the variants in SCLC development. We followed the ACMG guidelines that provide a systematic framework for interpretation of sequence variants to minimize instances of false positives. However, some of the variants could be incidental findings. For example, HOXB13 germline mutations are known to predispose to prostate cancers (40). To date, only one study showed enrichment of lung cancers in relatives of HOXB13 mutation carriers (41). We performed matched tumor sequencing, when possible, seeking evidence of functional consequences to further inform causality. Evidence of loss of the wild-type allele was observed in $21.4 \%$ of the germline-mutated cases with available tumor. Scarce tumor from the core needle biopsies limited our ability to investigate other mechanisms of wild-type allele silencing.

To validate our findings, we used the largest available SCLC cohort reported by George et al. (18), consisting almost exclusively of treatment-naïve and surgically resected SCLC. In contrast, our cohort consisted of patients who were seeking investigational systemic therapies at tumor recurrence and as such were enriched for patients who had received multiple prior therapies. Although the somatic mutational profiles of both cohorts were largely similar, our cohort had numerically lower frequency of TP53 and RB1 mutations compared with the George et al. cohort. It should be noted that only minority of patients with SCLC undergo surgical resection as reported by George et al. or receive multiple lines of therapies at recurrence as in our cohort. Despite notable differences between the two cohorts, we found a similar proportion of patients harboring pathogenic germline variants in both cohorts, suggesting that the findings at the germline level are generalizable. Additional studies are needed to reveal the interaction between genetic predisposition and tobacco exposure in SCLC development. Previous studies have shown that tobacco smoking increases lung cancer risk in germline TP53 mutation carriers (9). Our study implicates additional genes that may exacerbate SCLC risk in smokers, but these observations are inevitably tentative and further studies including linkage analyses will be needed for confirmation.

In conclusion, we demonstrate that SCLC, one of the most lethal cancers, thought to result almost exclusively from tobacco use, may have an inherited predisposition and reveal how differences in germline DNA may put some individuals at greater risk than others of developing SCLC. Together, these findings lay the foundation for understanding the interaction between genotype and tobacco exposure in exacerbating SCLC risk and could have substantial implications for targeted therapies and directed cancer screening for patients and their families.

\section{MATERIALS AND METHODS Study design}

We undertook a prospective study of heritable defects in cancer genes among patients with SCLC and EPSCC receiving care at the NCI using whole-exome sequencing of germline DNA. We validated our results in an independent SCLC cohort and compared our results with 53,105 cancer-free non-Finnish European individuals from the ExAC cohort. Germline genotypes were correlated with clinical outcomes. This was an observational study and thus did not involve randomization or blinding. 


\section{Enrollment of patients}

Patients with SCLC or EPSCC who were evaluated at the NCI Developmental Therapeutics Branch Clinics for treatment trials of relapsed SCNCs were offered participation in a study of the natural history of lung cancers (ClinicalTrials.gov \#NCT02146170; NCI protocol \#14-C-0105). Sixty-eight consecutive patients who enrolled on this study that offered germline sequencing and disclosure of results were included. Nineteen patients were identified using the National Institutes of Health Clinical Center's Biomedical Translational Research Information System (15). The NCI Laboratory of Pathology confirmed the diagnoses. Tumor pathologic characteristics are detailed in table S1. Patients were followed up by phone every 6 months until death or loss to follow-up. Human participants committee at NCI approved the studies; all patients provided written informed consent for germline and tumor sequencing.

Whole-exome data were generated from germline DNA isolated from peripheral blood mononuclear cells (PBMCs) for all patients. We sequenced the tumor exome and the RNA transcripts in selected cases. Clinical and demographic information were collected through the medical records and patient interviews. A detailed personal and family history of cancer and environmental exposure history were obtained in person using a standardized questionnaire (data file S2).

\section{Whole-exome sequencing}

DNA from macrodissected, formalin-fixed paraffin-embedded tumor and PBMCs was captured using Agilent SureSelect Clinical Research Exome kits (Agilent) and sequenced on Illumina NextSeq 500 sequencers (Illumina) according to the manufacturers' instructions. Germline variants were called using HaploType Caller 3.4.0, and only high-confidence variants (population variant frequency $\leq 1 \%$, sequencing depth $\geq 20$, Fisher score $<75$, and variant allele frequency (VAF) $\geq 25 \%$ ) were reported in this study.

Pathogenic germline variants from the published SCLC cohort (18) were identified using the Genome Analysis Toolkit (GATK) version 4.0.12 package (42) following the best practices $(43,44)$. Raw germline mutation calls were made using the HaplotypeCaller function in genomic variant call format (GVCF) mode. Raw calls were consolidated using the GenomicsDBImport function, followed by joint genotyping using the GenotypeGVCFs function. Single-nucleotide substitutions and indel variants were recalibrated using the VariantRecalibrator and ApplyVQSR functions with hg19 reference variant resource bundle downloaded from the GATK website. Called variants were functionally annotated using snpEff (version $4.3 \mathrm{t}$ ) (45). Functional impact of coding single-base substitutions was annotated using the dbNSFP (46) database (version 4.0b2c), followed by annotation of the ClinVar (47) database to identify known pathogenic and likely pathogenic variants.

Somatic variants were called using MuTect (version 1.1.7) (48) for single-nucleotide variants and Strelka (version 1.0.14) (49) for indels. Only high-confidence somatic variants (population frequency $\leq 1 \%$, sequencing depth $\geq 20 \times$ for both tumor and paired normal DNA, $\mathrm{VAF} \geq 10 \%$ ) were reported in this study. Variants were summarized and compared using maftools (version 2.4.0) (50). For each sample, TMB and mutational signatures were determined (25). LOH was analyzed on tumor samples and matched normal blood samples using Sequenza (version 3.0.0) (51). Zygosity of each pathogenic variant was inferred in the tumor according to its variant allele fraction.

\section{Predisposition genes selected for analysis}

A total of 607 genes were chosen for analysis on the basis of review of the ACMG gene list and the medical literature (fig. S1 and table S3) (52-54). These genes were classified according to six categories: autosomal dominant cancer predisposition genes, autosomal recessive cancer predisposition genes, oncogenes, tumor suppressor genes, cancer genes (53), and other. Among them, 55 genes were autosomal dominant cancer predisposition genes, 26 autosomal recessive cancer predisposition genes, 22 oncogenes, 23 tumor suppressor genes, and 118 cancer genes [somatic and germline catalogue of somatic mutations in cancer (COSMIC) Cancer Gene Census (53)]. Of the 607 genes, 134 were selected for evaluation on the basis of their recurrent somatic mutation in SCLC $(18,55,56)$. Among these 134 SCLC genes, $13 \%$ were cancer predisposition genes, $7 \%$ were oncogenes, $4 \%$ were tumor suppressor genes, and $23 \%$ cancer genes. Three hundred sixty three additional genes curated from previously published cancer predisposition genes $(19,57)$ were also added (fig. S1 and table S3).

\section{Germline data analysis}

The sequencing data were analyzed for the presence of single-nucleotide variants and small insertions and deletions (52). All variants with less than $1 \%$ frequency in the public database ExAC were interpreted (27). Variants with total coverage $>20 \times$ and variant allele frequency $($ VAF) $>0.25$ were included. Other variants were excluded. Nonsilent coding variants that passed quality-control and minor-allele population frequency checks were classified as pathogenic, likely pathogenic, VUS, likely benign, or benign. Classification criteria included information from curated databases, computational predictions of mutational effect on protein function, and ACMG guidelines for interpretation (20). The selection was supplemented on the basis of the medical literature and second hits identified in the tumor genome when available. VUS was reviewed but not reported in this analysis. Only pathogenic and likely pathogenic variants including nonsense, frameshift, splice site, and missense variants were included. For missense variants, only those with published evidence of being pathogenic or likely pathogenic in ClinVar (47) were included. The final analysis included cancer-related genes only. Moreover, only ACMG cancer-related genes were considered as actionable cancerrelated genes (21). For pathogenic and likely pathogenic variants, germline subpopulation frequencies were retrieved from the dbSNP allele frequency aggregator (ALFA) database (version 20200227123210) using the dbSNP rsid as reference. Variants lacking population frequencies from the ALFA database were retrieved from either Genome Aggregation Database or Population Architecture through Genomics and Environment (PAGE) studies using the dbSNP website (www. ncbi.nlm.nih.gov/snp/docs/gsr/alfa/). The details of the data analysis and interpretation are provided in Fig. 1 and fig. S2.

\section{Statistical analysis}

Fisher's exact tests and Wilcoxon rank-sum tests were used to test the difference between the two mutation groups with respect to dichotomous and continuous variables, respectively. Ordered categorical variables were compared between mutation groups using an exact Cochran-Armitage test for trend. Race categories were compared between mutation groups using Mehta's modification to Fisher's exact test. We used logistic regression and present ORs and 95\% CIs to assess associations between clinical characteristics and the presence of a pathogenic germline variant. Two-sided Fisher's exact tests were used to compare the frequencies of pathogenic 
germline variants in genes that were identified in our patients with noncancer population in ExAC. ORs and $P$ values reported for rare variant burden analysis were obtained from two-sided Fisher's exact tests to compare total burden of rare deleterious variants relative to rare synonymous variants in cases and controls. $P$ values $<0.05$ were considered significant. RFS and OS curves were created by the Kaplan-Meier method and compared with log-rank tests. RFS was determined from the date of completion of first-line platinum-based chemotherapy until date of relapse or last follow-up without relapse, whereas OS was determined from date of diagnosis until date of death or last follow-up. Multivariable Cox proportional hazards models were created to assess the effect of pathogenic variants after adjusting for known confounders. HRs were computed from Cox models, and $P$ values presented along with HRs are for a test of whether the $\mathrm{HR}=1.0$. Statistical analyses were performed with PRISM software version 8.1.2, R version 1.2.135 (R Foundation for Statistical Computing), STATA software version 16.0 (Stata-Corp), and SAS version 9.4 (SAS Institute Inc.). All statistical tests were two-sided.

\section{SUPPLEMENTARY MATERIALS}

\section{stm.sciencemag.org/cgi/content/full/13/578/eabc7488/DC1}

Fig. S1. Detail and categories of the 607 genes analyzed for germline mutations. Fig. S2. Overview of filter-based strategy to identify candidate SCLC susceptibility genes. Fig. S3. SCNC germline mutation characteristics.

Fig. S4. SCLC germline mutation characteristics according to validated categories. Fig. S5. MLH1 pathogenic variant at RNA level.

Fig. S6. Tumor characteristics according to germline alteration status.

Fig. S7. Common somatic mutations in our cohort and an independent cohort.

Fig. S8. Common likely pathogenic genes and variants between $\mathrm{NCl}$ cohort and George et al. cohort.

Fig. S9. Kaplan-Meier curves of RFS and OS in patients with limited-stage and extensive-stage SCLC.

Fig. S10. Clinical characteristics and outcomes of patients stratified by pathogenic germline variants in known cancer predisposition genes (BROCAv10 panel).

Fig. S11. Clinical characteristics and outcomes of patients stratified by ACMG pathogenic germline variants.

Data file S1. Supplementary tables S1 to S9:

Table S1. Patient tumor pathological characteristics.

Table S2. Sequencing characteristics.

Table S3. Gene list used for variant annotation.

Table S4. Molecular characteristics of germline mutations in patients with SCNC.

Table S5. Purity and ploidy of tumor samples.

Table S6. Characteristics of germline mutations in George et al. cohort patients.

Table S7. Minor allele frequencies of pathogenic variants by population.

Table S8. Clinical characteristics of patients with SCLC.

Table S9. Systemic treatments received by patients.

Data file S2. Family history and environmental exposure questionnaires.

View/request a protocol for this paper from Bio-protocol.

\section{REFERENCES AND NOTES}

1. F. Bray, J. Ferlay, I. Soerjomataram, R. L. Siegel, L. A. Torre, A. Jemal, Global cancer statistics 2018: GLOBOCAN estimates of incidence and mortality worldwide for 36 cancers in 185 countries. CA Cancer J. Clin. 68, 394-424 (2018).

2. Y. Bosse, C. I. Amos, A decade of GWAS results in lung cancer. Cancer Epidemiol. Biomarkers Prev. 27, 363-379 (2018).

3. R. Govindan, N. Page, D. Morgensztern, W. Read, R. Tierney, A. Vlahiotis, E. L. Spitznagel, J. Piccirillo, Changing epidemiology of small-cell lung cancer in the United States over the last 30 years: Analysis of the surveillance, epidemiologic, and end results database. J. Clin. Oncol. 24, 4539-4544 (2006).

4. S. A. Kenfield, E. K. Wei, M. J. Stampfer, B. A. Rosner, G. A. Colditz, Comparison of aspects of smoking among the four histological types of lung cancer. Tob. Control 17, 198-204 (2008).

5. S. C. Wang, J. J. Tang, T. T. Sun, X. B. Zheng, J. Li, H. L. Sun, X. L. Zhou, C. L. Zhou, H. Y. Zhang, Z. B. Cheng, H. Q. Ma, H. H. Sun, Survival changes in patients with small cell lung cancer and disparities between different sexes, socioeconomic statuses and ages. Sci. Rep. 7, 1339 (2017).
6. A. Thomas, P. Pattanayak, E. Szabo, P. Pinsky, Characteristics and outcomes of small cell lung cancer detected by CT screening. Chest 154, 1284-1290 (2018).

7. A. Thomas, I. Mian, C. Tlemsani, L. Pongor, N. Takahashi, K. Maignan, J. Snider, G. Li, G. Frampton, S. Ali, S. Kim, S. Nichols, V. Rajapakse, U. Guha, E. Sharon, J. Fujimoto, C. A. Moran, I. I. Wistuba, J. S. Wei, J. Khan, E. Szabo, A. Z. Torres, K. R. Carson, Clinical and genomic characteristics of small cell lung cancer in never smokers: Results from a retrospective multicenter cohort study. Chest 158, 1723-1733 (2020).

8. S.-H. Ou, A. Ziogas, J. A. Zell, Prognostic factors for survival in extensive stage small cell lung cancer (ED-SCLC): The importance of smoking history, socioeconomic and marital statuses, and ethnicity. J. Thorac. Oncol. 4, 37-43 (2009).

9. S.-J. Hwang, L. S.-C. Cheng, G. Lozano, C. I. Amos, X. Gu, L. C. Strong, Lung cancer risk in germline $\mathrm{p} 53$ mutation carriers: Association between an inherited cancer predisposition, cigarette smoking, and cancer risk. Hum. Genet. 113, 238-243 (2003).

10. R. A. Kleinerman, R. E. Tarone, D. H. Abramson, J. M. Seddon, F. P. Li, M. A. Tucker, Hereditary retinoblastoma and risk of lung cancer. J. Natl. Cancer Inst. 92, 2037-2039 (2000).

11. T. Rafnar, G. R. Sigurjonsdottir, S. N. Stacey, G. Halldorsson, P. Sulem, L. M. Pardo, H. Helgason, S. T. Sigurdsson, T. Gudjonsson, L. Tryggvadottir, G. H. Olafsdottir, J. G. Jonasson, K. Alexiusdottir, A. Sigurdsson, J. Gudmundsson, J. Saemundsdottir, J. K. Sigurdsson, H. Johannsdottir, A. Uitterlinden, S. H. Vermeulen, T. E. Galesloot, D. C. Allain, M. Lacko, B. Sigurgeirsson, K. Thorisdottir, O. T. Johannsson, F. Sigurdsson, G. B. Ragnarsson, H. Isaksson, H. Hardardottir, T. Gudbjartsson, D. F. Gudbjartsson, G. Masson, L. Kiemeney, A. E. Toland, T. Nijsten, W. H. M. Peters, J. H. Olafsson, S. Jonsson, U. Thorsteinsdottir, G. Thorleifsson, K. Stefansson, Association of BRCA2 K3326* with small cell lung cancer and squamous cell cancer of the skin. J. Natl. Cancer Inst. 110, 967-974 (2018).

12. K. L. Huang, R. J. Mashl, Y. Wu, D. I. Ritter, J. Wang, C. Oh, M. Paczkowska, S. Reynolds, M. A. Wyczalkowski, N. Oak, A. D. Scott, M. Krassowski, A. D. Cherniack, K. E. Houlahan, R. Jayasinghe, L. B. Wang, D. C. Zhou, D. Liu, S. Cao, Y. W. Kim, A. Koire, J. F. McMichael, V. Hucthagowder, T. B. Kim, A. Hahn, C. Wang, M. D. McLellan, F. Al-Mulla, K. J. Johnson; Cancer Genome Atlas Research Network, O. Lichtarge, P. C. Boutros, B. Raphael, A. J. Lazar, W. Zhang, M. C. Wendl, R. Govindan, S. Jain, D. Wheeler, S. Kulkarni, J. F. Dipersio, J. Reimand, F. Meric-Bernstam, K. Chen, I. Shmulevich, S. E. Plon, F. Chen, L. Ding, Pathogenic germline variants in 10,389 adult cancers. Cell 173, 355-370.e14 (2018).

13. X. Zheng, D. Liu, J. T. Fallon, M. Zhong, Distinct genetic alterations in small cell carcinoma from different anatomic sites. Exp. Hematol. Oncol. 4, 2 (2015).

14. N. G. Balanis, K. M. Sheu, F. N. Esedebe, S. J. Patel, B. A. Smith, J. W. Park, S. Alhani, B. N. Gomperts, J. Huang, O. N. Witte, T. G. Graeber, Pan-cancer convergence to a small-cell neuroendocrine phenotype that shares susceptibilities with hematological malignancies. Cancer Cell 36, 17-34.e7 (2019).

15. J. J. Cimino, E. J. Ayres, L. Remennik, S. Rath, R. Freedman, A. Beri, Y. Chen, V. Huser, The National Institutes of Health's Biomedical Translational Research Information System (BTRIS): Design, contents, functionality and experience to date. J. Biomed. Inform. 52, 11-27 (2014).

16. A. Ardizzoni, M. Tiseo, L. Boni, Validation of standard definition of sensitive versus refractory relapsed small cell lung cancer: A pooled analysis of topotecan second-line trials. Eur. J. Cancer 50, 2211-2218 (2014).

17. J. G. Tate, S. Bamford, H. C. Jubb, Z. Sondka, D. M. Beare, N. Bindal, H. Boutselakis, C. G. Cole, C. Creatore, E. Dawson, P. Fish, B. Harsha, C. Hathaway, S. C. Jupe, C. Y. Kok, K. Noble, L. Ponting, C. C. Ramshaw, C. E. Rye, H. E. Speedy, R. Stefancsik, S. L. Thompson, S. Wang, S. Ward, P. J. Campbell, S. A. Forbes, COSMIC: The catalogue of somatic mutations in cancer. Nucleic Acids Res. 47, D941-D947 (2019).

18. J. George, J. S. Lim, S. J. Jang, Y. Cun, L. Ozretić, G. Kong, F. Leenders, X. Lu, L. Fernández-Cuesta, G. Bosco, C. Müller, I. Dahmen, N. S. Jahchan, K.-S. Park, D. Yang, A. N. Karnezis, D. Vaka, A. Torres, M. S. Wang, J. O. Korbel, R. Menon, S.-M. Chun, D. Kim, M. Wilkerson, N. Hayes, D. Engelmann, B. Pützer, M. Bos, S. Michels, I. Vlasic, D. Seidel, B. Pinther, P. Schaub, C. Becker, J. Altmüller, J. Yokota, T. Kohno, R. Iwakawa, K. Tsuta, M. Noguchi, T. Muley, H. Hoffmann, P. A. Schnabel, I. Petersen, Y. Chen, A. Soltermann, V. Tischler, C.-m. Choi, Y.-H. Kim, P. P. Massion, Y. Zou, D. Jovanovic, M. Kontic, G. M. Wright, P. A. Russell, B. Solomon, I. Koch, M. Lindner, L. A. Muscarella, A. la Torre, J. K. Field, M. Jakopovic, J. Knezevic, E. Castaños-Vélez, L. Roz, U. Pastorino, O.-T. Brustugun, M. Lund-lversen, E. Thunnissen, J. Köhler, M. Schuler, J. Botling, M. Sandelin, M. Sanchez-Cespedes, H. B. Salvesen, V. Achter, U. Lang, M. Bogus, P. M. Schneider, T. Zander, S. Ansén, M. Hallek, J. Wolf, M. Vingron, Y. Yatabe, W. D. Travis, P. Nürnberg, C. Reinhardt, S. Perner, L. Heukamp, R. Büttner, S. A. Haas, E. Brambilla, M. Peifer, J. Sage, R. K. Thomas, Comprehensive genomic profiles of small cell lung cancer. Nature 524, 47-53 (2015).

19. N. Rahman, Realizing the promise of cancer predisposition genes. Nature 505, 302-308 (2014).

20. S. Richards, N. Aziz, S. Bale, D. Bick, S. Das, J. Gastier-Foster, W. W. Grody, M. Hegde, E. Lyon, E. Spector, K. Voelkerding, H. L. Rehm; ACMG Laboratory Quality Assurance Committee, Standards and guidelines for the interpretation of sequence variants: A joint 
consensus recommendation of the American College of Medical Genetics and Genomics and the Association for Molecular Pathology. Genet. Med. 17, 405-424 (2015).

21. S. S. Kalia, K. Adelman, S. J. Bale, W. K. Chung, C. Eng, J. P. Evans, G. E. Herman, S. B. Hufnagel, T. E. Klein, B. R. Korf, K. D. McKelvey, K. E. Ormond, C. S. Richards, C. N. Vlangos, M. Watson, C. L. Martin, D. T. Miller, Recommendations for reporting of secondary findings in clinical exome and genome sequencing, 2016 update (ACMG SF v2.0): A policy statement of the American College of Medical Genetics and Genomics. Genet. Med. 19, 249-255 (2017).

22. B. H. Shirts, S. Casadei, A. L. Jacobson, M. K. Lee, S. Gulsuner, R. L. Bennett, M. Miller, S. A. Hall, H. Hampel, F. M. Hisama, L. V. Naylor, C. Goetsch, K. Leppig, J. F. Tait, S. M. Scroggins, E. H. Turner, R. Livingston, S. J. Salipante, M. C. King, T. Walsh, C. C. Pritchard, Improving performance of multigene panels for genomic analysis of cancer predisposition. Genet. Med. 18, 974-981 (2016).

23. Y. D. Connor, D. Miao, D. I. Lin, C. Hayne, B. E. Howitt, J. L. Dalrymple, K. R. DeLeonardis, M. R. Hacker, K. M. Esselen, M. Shea, Germline mutations of SMARCA4 in small cell carcinoma of the ovary, hypercalcemic type and in SMARCA4-deficient undifferentiated uterine sarcoma: Clinical features of a single family and comparison of large cohorts. Gynecol. Oncol. 157, 106-114 (2019).

24. B. Niu, K. Ye, Q. Zhang, C. Lu, M. Xie, M. D. McLellan, M. C. Wendl, L. Ding, MSIsensor: Microsatellite instability detection using paired tumor-normal sequence data. Bioinformatics 30, 1015-1016 (2014).

25. L. B. Alexandrov, S. Nik-Zainal, D. C. Wedge, S. A. J. R. Aparicio, S. Behjati, A. V. Biankin, G. R. Bignell, N. Bolli, A. Borg, A.-L. Børresen-Dale, S. Boyault, B. Burkhardt, A. P. Butler, C. Caldas, H. R. Davies, C. Desmedt, R. Eils, J. E. Eyfjörd, J. A. Foekens, M. Greaves, F. Hosoda, B. Hutter, T. llicic, S. Imbeaud, M. Imielinski, N. Jäger, D. T. W. Jones, D. Jones, S. Knappskog, M. Kool, S. R. Lakhani, C. López-Otín, S. Martin, N. C. Munshi, H. Nakamura, P. A. Northcott, M. Pajic, E. Papaemmanuil, A. Paradiso, J. V. Pearson, X. S. Puente, K. Raine, M. Ramakrishna, A. L. Richardson, J. Richter, P. Rosenstiel, M. Schlesner, T. N. Schumacher, P. N. Span, J. W. Teague, Y. Totoki, A. N. J. Tutt, R. Valdés-Mas, M. M. van Buuren, L. van 't Veer, A. Vincent-Salomon, N. Waddell, L. R. Yates; Australian Pancreatic Cancer Genome Initiatives; ICGC Breast Cancer Consortium; ICGC MMML-Seq Consortium; ICGC PedBrain, J. Zucman-Rossi, P. A. Futreal, U. McDermott, P. Lichter, M. Meyerson, S. M. Grimmond, R. Siebert, E. Campo, T. Shibata, S. M. Pfister, P. J. Campbell, M. R. Stratton, Signatures of mutational processes in human cancer. Nature 500, 415-421 (2013).

26. A. G. Knudson, Two genetic hits (more or less) to cancer. Nat. Rev. Cancer 1, 157-162 (2001).

27. M. Lek, K. J. Karczewski, E. V. Minikel, K. E. Samocha, E. Banks, T. Fennell, A. H. O'Donnell-Luria, J. S. Ware, A. J. Hill, B. B. Cummings, T. Tukiainen, D. P. Birnbaum, J. A. Kosmicki, L. E. Duncan, K. Estrada, F. Zhao, J. Zou, E. Pierce-Hoffman, J. Berghout, D. N. Cooper, N. Deflaux, M. DePristo, R. Do, J. Flannick, M. Fromer, L. Gauthier, J. Goldstein, N. Gupta, D. Howrigan, A. Kiezun, M. I. Kurki, A. L. Moonshine, P. Natarajan, L. Orozco, G. M. Peloso, R. Poplin, M. A. Rivas, V. Ruano-Rubio, S. A. Rose, D. M. Ruderfer, K. Shakir, P. D. Stenson, C. Stevens, B. P. Thomas, G. Tiao, M. T. Tusie-Luna, B. Weisburd, H.-H. Won, D. Yu, D. M. Altshuler, D. Ardissino, M. Boehnke, J. Danesh, S. Donnelly, R. Elosua, J. C. Florez, S. B. Gabriel, G. Getz, S. J. Glatt, C. M. Hultman, S. Kathiresan, M. Laakso, S. McCarroll, M. I. McCarthy, D. McGovern, R. McPherson, B. M. Neale, A. Palotie, S. M. Purcell, D. Saleheen, J. M. Scharf, P. Sklar, P. F. Sullivan, J. Tuomilehto, M. T. Tsuang, H. C. Watkins, J. G. Wilson, M. J. Daly, D. G. MacArthur; Exome Aggregation Consortium, Analysis of protein-coding genetic variation in 60,706 humans. Nature 536, 285-291 (2016).

28. E. Markkanen, J. Dorn, U. Hübscher, MUTYH DNA glycosylase: The rationale for removing undamaged bases from the DNA. Front. Genet. 4, 18 (2013).

29. V. Ruggieri, E. Pin, M. T. Russo, F. Barone, P. Degan, M. Sanchez, M. Quaia, A. Minoprio, E. Turco, F. Mazzei, A. Viel, M. Bignami, Loss of MUTYH function in human cells leads to accumulation of oxidative damage and genetic instability. Oncogene 32, 4500-4508 (2013).

30. G. Rennert, F. Lejbkowicz, I. Cohen, M. Pinchev, H. S. Rennert, O. Barnett-Griness, MutYH mutation carriers have increased breast cancer risk. Cancer 118, 1989-1993 (2012).

31. J. Balmaña, F. Balaguer, A. Cervantes, D. Arnold; ESMO Guidelines Working Group, Familial risk-colorectal cancer: ESMO clinical practice guidelines. Ann. Oncol. 24 (Suppl. 6), vi73-vi80 (2013).

32. T. Rafnar, D. F. Gudbjartsson, P. Sulem, A. Jonasdottir, A. Sigurdsson, A. Jonasdottir, S. Besenbacher, P. Lundin, S. N. Stacey, J. Gudmundsson, O. T. Magnusson, L. le Roux, G. Orlygsdottir, H. T. Helgadottir, H. Johannsdottir, A. Gylfason, L. Tryggvadottir, J. G. Jonasson, A. de Juan, E. Ortega, J. M. Ramon-Cajal, M. D. Garcia-Prats, C. Mayordomo, A. Panadero, F. Rivera, K. K. Aben, A. M. van Altena, L. F. Massuger, M. Aavikko, P. M. Kujala, S. Staff, L. A. Aaltonen, K. Olafsdottir, J. Bjornsson, A. Kong, A. Salvarsdottir, H. Saemundsson, K. Olafsson, K. R. Benediktsdottir, J. Gulcher, G. Masson, L. A. Kiemeney, J. I. Mayordomo, U. Thorsteinsdottir, K. Stefansson, Mutations in BRIP1 confer high risk of ovarian cancer. Nat. Genet. 43, 1104-1107 (2011).

33. A. Thomas, Y. Pommier, Small cell lung cancer: Time to revisit DNA-damaging chemotherapy. Sci. Transl. Med. 8, 346fs12 (2016).
34. H. Farmer, N. McCabe, C. J. Lord, A. N. J. Tutt, D. A. Johnson, T. B. Richardson, M. Santarosa, K. J. Dillon, I. Hickson, C. Knights, N. M. B. Martin, S. P. Jackson, G. C. M. Smith, A. Ashworth Targeting the DNA repair defect in BRCA mutant cells as a therapeutic strategy. Nature 434, 917-921 (2005)

35. H. E. Bryant, N. Schultz, H. D. Thomas, K. M. Parker, D. Flower, E. Lopez, S. Kyle, M. Meuth N. J. Curtin, T. Helleday, Specific killing of BRCA2-deficient tumours with inhibitors of poly(ADP-ribose) polymerase. Nature 434, 913-917 (2005).

36. W. K. Eng, L. Faucette, R. K. Johnson, R. Sternglanz, Evidence that DNA topoisomerase-I is necessary for the cyto-toxic effects of camptothecin. Mol. Pharmacol. 34, 755-760 (1988).

37. J. Nitiss, J. C. Wang, DNA topoisomerase-targeting antitumor drugs can be studied in yeast. Proc. Natl. Acad. Sci. U.S.A. 85, 7501-7505 (1988).

38. Y. Shen, F. L. Rehman, Y. Feng, J. Boshuizen, I. Bajrami, R. Elliott, B. Wang, C. J. Lord, L. E. Post, A. Ashworth, BMN 673, a novel and highly potent PARP1/2 inhibitor for the treatment of human cancers with DNA repair deficiency. Clin. Cancer Res. 19, 5003-5015 (2013).

39. J. Mateo, C. J. Lord, V. Serra, A. Tutt, J. Balmana, M. Castroviejo-Bermejo, C. Cruz, A. Oaknin, S. B. Kaye, J. S. de Bono, A decade of clinical development of PARP inhibitors in perspective. Ann. Oncol. 30, 1437-1447 (2019).

40. C. M. Ewing, A. M. Ray, E. M. Lange, K. A. Zuhlke, C. M. Robbins, W. D. Tembe, K. E. Wiley, S. D. Isaacs, D. Johng, Y. Wang, C. Bizon, G. Yan, M. Gielzak, A. W. Partin, V. Shanmugam, T. Izatt, S. Sinari, D. W. Craig, S. L. Zheng, P. C. Walsh, J. E. Montie, J. Xu, J. D. Carpten, W. B. Isaacs, K. A. Cooney, Germline mutations in HOXB13 and prostate-cancer risk. N. Engl. J. Med. 366, 141-149 (2012).

41. Y. Momozawa, Y. Iwasaki, M. Hirata, X. Liu, Y. Kamatani, A. Takahashi, K. Sugano, T. Yoshida, Y. Murakami, K. Matsuda, H. Nakagawa, A. B. Spurdle, M. Kubo, Germline pathogenic variants in 7636 Japanese patients with prostate cancer and 12366 CONTROLS. J. Natl. Cancer Inst. 112, 369-376 (2020).

42. A. McKenna, M. Hanna, E. Banks, A. Sivachenko, K. Cibulskis, A. Kernytsky, K. Garimella, D. Altshuler, S. Gabriel, M. Daly, M. A. DePristo, The genome analysis toolkit: A MapReduce framework for analyzing next-generation DNA sequencing data. Genome Res. 20, 1297-1303 (2010).

43. M. A. DePristo, E. Banks, R. Poplin, K. V. Garimella, J. R. Maguire, C. Hartl, A. A. Philippakis, G. del Angel, M. A. Rivas, M. Hanna, A. McKenna, T. J. Fennell, A. M. Kernytsky, A. Y. Sivachenko, K. Cibulskis, S. B. Gabriel, D. Altshuler, M. J. Daly, A framework for variation discovery and genotyping using next-generation DNA sequencing data. Nat. Genet. 43, 491-498 (2011).

44. G. A. Van der Auwera, M. O. Carneiro, C. Hartl, R. Poplin, G. Del Angel, A. Levy-Moonshine, T. Jordan, K. Shakir, D. Roazen, J. Thibault, E. Banks, K. V. Garimella, D. Altshuler, S. Gabriel, M. A. DePristo, From FastQ data to high confidence variant calls: The Genome Analysis Toolkit best practices pipeline. Curr. Protoc. Bioinformatics 43, 11.10.11-11.10.33 (2013).

45. P. Cingolani, A. Platts, L. le Wang, M. Coon, T. Nguyen, L. Wang, S. J. Land, X. Lu, D. M. Ruden, A program for annotating and predicting the effects of single nucleotide polymorphisms, SnpEff: SNPs in the genome of Drosophila melanogaster strain w1118; iso-2; iso-3. Fly 6, 80-92 (2012).

46. X. Liu, C. Wu, C. Li, E. Boerwinkle, dbNSFP v3.0: A one-stop database of functional predictions and annotations for human nonsynonymous and splice-site SNVs. Hum. Mutat. 37, 235-241 (2016).

47. M. J. Landrum, J. M. Lee, M. Benson, G. R. Brown, C. Chao, S. Chitipiralla, B. Gu, J. Hart, D. Hoffman, W. Jang, K. Karapetyan, K. Katz, C. Liu, Z. Maddipatla, A. Malheiro, K. McDaniel, M. Ovetsky, G. Riley, G. Zhou, J. B. Holmes, B. L. Kattman, D. R. Maglott, ClinVar: Improving access to variant interpretations and supporting evidence. Nucleic Acids Res. 46, D1062-D1067 (2018).

48. K. Cibulskis, M. S. Lawrence, S. L. Carter, A. Sivachenko, D. Jaffe, C. Sougnez, S. Gabriel, M. Meyerson, E. S. Lander, G. Getz, Sensitive detection of somatic point mutations in impure and heterogeneous cancer samples. Nat. Biotechnol. 31, 213-219 (2013).

49. S. Kim, K. Scheffler, A. L. Halpern, M. A. Bekritsky, E. Noh, M. Kallberg, X. Chen, Y. Kim, D. Beyter, P. Krusche, C. T. Saunders, Strelka2: Fast and accurate calling of germline and somatic variants. Nat. Methods 15, 591-594 (2018).

50. A. Mayakonda, D. C. Lin, Y. Assenov, C. Plass, H. P. Koeffler, Maftools: Efficient and comprehensive analysis of somatic variants in cancer. Genome Res. 28, 1747-1756 (2018).

51. F. Favero, T. Joshi, A. M. Marquard, N. J. Birkbak, M. Krzystanek, Q. Li, Z. Szallasi, A. C. Eklund, Sequenza: Allele-specific copy number and mutation profiles from tumor sequencing data. Ann. Oncol. 26, 64-70 (2015).

52. A. S. Brohl, R. Patidar, C. E. Turner, X. Wen, Y. K. Song, J. S. Wei, K. A. Calzone, J. Khan, Frequent inactivating germline mutations in DNA repair genes in patients with Ewing sarcoma. Genet. Med. 19, 955-958 (2017).

53. Z. Sondka, S. Bamford, C. G. Cole, S. A. Ward, I. Dunham, S. A. Forbes, The COSMIC Cancer Gene Census: Describing genetic dysfunction across all human cancers. Nat. Rev. Cancer 18, 696-705 (2018). 
54. J. Zhang, M. F. Walsh, G. Wu, M. N. Edmonson, T. A. Gruber, J. Easton, D. Hedges, X. Ma, X. Zhou, D. A. Yergeau, M. R. Wilkinson, B. Vadodaria, X. Chen, R. B. McGee, S. Hines-Dowell, R. Nuccio, E. Quinn, S. A. Shurtleff, M. Rusch, A. Patel, J. B. Becksfort, S. Wang, M. S. Weaver, L. Ding, E. R. Mardis, R. K. Wilson, A. Gajjar, D. W. Ellison, A. S. Pappo, C.-H. Pui, K. E. Nichols, J. R. Downing, Germline mutations in predisposition genes in pediatric cancer. N. Engl. J. Med. 373, 2336-2346 (2015).

55. S. Umemura, S. Mimaki, H. Makinoshima, S. Tada, G. Ishii, H. Ohmatsu, S. Niho, K. Yoh, S. Matsumoto, A. Takahashi, M. Morise, Y. Nakamura, A. Ochiai, K. Nagai, R. Iwakawa, T. Kohno, J. Yokota, Y. Ohe, H. Esumi, K. Tsuchihara, K. Goto, Therapeutic priority of the $\mathrm{PI3K} / \mathrm{AKT} / \mathrm{mTOR}$ pathway in small cell lung cancers as revealed by a comprehensive genomic analysis. J. Thorac. Oncol. 9, 1324-1331 (2014).

56. C. M. Rudin, S. Durinck, E. W. Stawiski, J. T. Poirier, Z. Modrusan, D. S. Shames, E. A. Bergbower, Y. Guan, J. Shin, J. Guillory, C. S. Rivers, C. K. Foo, D. Bhatt, J. Stinson, F. Gnad, P. M. Haverty, R. Gentleman, S. Chaudhuri, V. Janakiraman, B. S. Jaiswal, C. Parikh, W. Yuan, Z. Zhang, H. Koeppen, T. D. Wu, H. M. Stern, R. L. Yauch, K. E. Huffman, D. D. Paskulin, P. B. Illei, M. Varella-Garcia, A. F. Gazdar, F. J. de Sauvage, R. Bourgon, J. D. Minna, M. V. Brock, S. Seshagiri, Comprehensive genomic analysis identifies SOX2 as a frequently amplified gene in small-cell lung cancer. Nat. Genet. 44, 1111-1116 (2012)

57. T. A. Knijnenburg, L. H. Wang, M. T. Zimmermann, N. Chambwe, G. F. Gao, A. D. Cherniack, H. H. Fan, H. Shen, G. P. Way, C. S. Greene, Y. X. Liu, R. Akbani, B. Feng L. A. Donehower, C. Miller, Y. Shen, M. Karimi, H. R. Chen, P. Kim, P. L. Jia, E. Shinbrot, S. J. Zhang, J. F. Liu, H. Hu, M. H. Bailey, C. Yau, D. Wolf, Z. M. Zhao, J. N. Weinstein, L. Li, L. Ding, G. B. Mills, P. W. Laird, D. A. Wheeler, I. Shmulevich; Cancer Genome Atlas Research Network, R. J. Monnat, Y. H. Xiao, C. Wang, Genomic and molecular landscape of DNA damage repair deficiency across the cancer genome atlas. Cell Rep. $\mathbf{2 3}$ 239-254.e6 (2018).
Acknowledgments: We gratefully acknowledge patients who volunteered to participate in the study and agreed to provide research biopsies. This study used the high-performance computational capabilities of the Biowulf Linux cluster at the NIH (https://hpc.nih.gov/). NLG207 and olaparib were supplied under a Collaborative Research and Development Agreement between the NCl, Ellipses Pharma, and AstraZeneca. Funding: This research was supported by the NIH Intramural Research Program, Center for Cancer Research, $\mathrm{NCl}$ at the $\mathrm{NIH}$ (ZIA BC 011793 to A.T.). Author contributions: Conceptualization, methodology, software, investigation, and writing original draft: A.T., C.T., N.T., and L.P. Conceptualization, methodology, software, and writing (review and editing): J.K., C.E.T., and J.S.W. Investigation and writing (review and editing): V.N.R., M.T., X.W., G.-A.F., K.T.S., P.D., C.K., A.R., S.S., L.S., R.V., S.W., S.N., W.D.F., Y.P., K.C., S.M.S., and U.G. Competing interests: A.T. and Y.P. report grants from AstraZeneca, Ellipses Pharma, EMD Serono, and Tarveda to the $\mathrm{NCI} / \mathrm{NIH}$ during the conduct of the study. U.G. received research funding from AstraZeneca and Aurigene, had Clinical Trial Agreement with AstraZeneca, and is currently an employee of Bristol-Myers Squibb. C.K. serves on the advisory board of Novartis. All other authors declare that they have no competing interests. Data and materials availability: All data associated with this study are present in the paper or the Supplementary Materials.

Submitted 11 May 2020

Resubmitted 27 July 2020

Accepted 6 January 2021

Published 27 January 202

10.1126/scitranslmed.abc7488

Citation: C. Tlemsani, N. Takahashi, L. Pongor, V. N. Rajapakse, M. Tyagi, X. Wen, G.-A. Fasaye K. T. Schmidt, P. Desai, C. Kim, A. Rajan, S. Swift, L. Sciuto, R. Vilimas, S. Webb, S. Nichols, W. D. Figg Y. Pommier, K. Calzone, S. M. Steinberg, J. S. Wei, U. Guha, C. E. Turner, J. Khan, A. Thomas, Whole-exome sequencing reveals germline-mutated small cell lung cancer subtype with favorable response to DNA repair-targeted therapies. Sci. Transl. Med. 13, eabc7488 (2021). 